\title{
THE POPULATION OF HIGH-REDSHIFT ACTIVE GALACTIC NUCLEI IN THE CHANDRA-COSMOS SURVEY
}

\author{
F. Civano ${ }^{1}$, M. Brusa ${ }^{2}$, A. Comastri ${ }^{3}$, M. Elvis ${ }^{1}$, M. Salvato ${ }^{4}$, G. Zamorani ${ }^{3}$, P. Capak $^{5}$, F. Fiore ${ }^{6}$, R. Gilli ${ }^{3}$, H. Hao ${ }^{1}$,

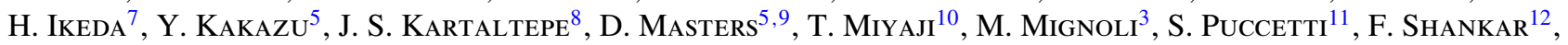 \\ J. Silverman ${ }^{13}$, C. Vignali ${ }^{14}$, A. ZeZas ${ }^{1,15}$, AND A. M. KoeKemoer ${ }^{16}$ \\ ${ }^{1}$ Harvard Smithsonian Center for Astrophysics, 60 Garden Street, Cambridge, MA 02138, USA \\ ${ }^{2}$ Max Planck Institut für Extraterrestrische Physik Giessenbach strasse 1, D-85748 Garching, Germany \\ ${ }^{3}$ INAF-Osservatorio Astronomico di Bologna, via Ranzani 1, I-40127 Bologna, Italy \\ ${ }^{4}$ Max-Planck-Institute for Plasma Physics, Boltzmannstrass 2, Garching D-85748, Germany \\ ${ }^{5}$ California Institute of Technology, 1201 East California Boulevard, Pasadena, CA 91125, USA \\ ${ }^{6}$ INAF-Osservatorio Astronomico di Roma, via Frascati 33, Monteporzio-Catone (Roma) I-00040, Italy \\ ${ }^{7}$ Graduate School of Science and Engineering, Ehime University, 2-5 Bunkyo-cho, Matsuyama 790-8577, Japan \\ ${ }^{8}$ Institute for Astronomy, University of Hawaii, 2680 Woodlawn Drive, Honolulu, HI 96822, USA \\ ${ }^{9}$ Department of Physics and Astronomy, University of California, 900 University Avenue, Riverside, CA 92521, USA \\ ${ }^{10}$ Instituto de Astronomia, Universidad Nacional Autonoma de Mexico-Ensenada, Km. 103 Carretera Tijuana-Ensenada, 22860 Ensenada, Mexico \\ ${ }^{11}$ ASI Science Data Center, via Galileo Galilei, 00044 Frascati, Italy \\ ${ }^{12}$ Max-Planck-Institut fü̈ Astrophysik, Karl-Schwarzschild-Str. 1, D-85748 Garching, Germany \\ ${ }^{13}$ Institute for the Physics and Mathematics of the Universe (IPMU), University of Tokyo, Kashiwanoha 5-1-5, \\ Kashiwa, Chiba 277-8568, Japan \\ ${ }^{14}$ Dipartimento di Astronomia, Universitá di Bologna, via Ranzani 1, 40127 Bologna, Italy \\ 15 Physics Department, University of Crete, P.O. Box 2208, GR-710 03 Heraklion, Crete, Greece \\ ${ }^{16}$ Space Telescope Science Institute, 3700 San Martin Drive, Baltimore, MD 21218, USA \\ Received 2010 December 23; accepted 2011 August 8; published 2011 October 21
}

\begin{abstract}
We present the high-redshift $(3<z<5.3) 0.5-2 \mathrm{keV}$ number counts and the $2-10 \mathrm{keV}$ (rest-frame) space density of X-ray-selected active galactic nuclei (AGNs) detected in the Chandra Cosmic Evolution Survey. The sample comprises 81 X-ray-detected sources with available spectroscopic (31) and photometric (50) redshifts plus 20 sources with a formal $z_{\text {phot }}<3$ but with a broad photometric redshift probability distribution, such that $z_{\text {phot }}+1 \sigma>3$. Eighty-one sources are selected in the $0.5-2 \mathrm{keV}$ band, fourteen are selected in the $2-10 \mathrm{keV}$ and six in the $0.5-10 \mathrm{keV}$ bands. We sample the high-luminosity $\left(\log L_{(2-10 \mathrm{keV})}>44.15 \mathrm{erg} \mathrm{s}^{-1}\right)$ space density up to $z \sim 5$ and a fainter luminosity range $\left(43.5 \mathrm{erg} \mathrm{s}^{-1}<\log L_{(2-10 \mathrm{keV})}<44.15 \mathrm{erg} \mathrm{s}^{-1}\right)$ than previous studies, up to $z=3.5$. We weighted the contribution to the number counts and the space density of the sources with photometric redshift by using their probability of being at $z>3$. We find that the space density of high-luminosity AGNs declines exponentially at all the redshifts, confirming the trend observed for optically selected quasars. At lower luminosity, the measured space density is not conclusive, and a larger sample of faint sources is needed. Comparisons with optical luminosity functions and black hole formation models are presented together with prospects for future surveys.
\end{abstract}

Key words: evolution - galaxies: active - surveys - X-rays: galaxies

Online-only material: color figures

\section{INTRODUCTION}

To properly test models on the co-evolution of black holes (BHs) and galaxies (e.g., Granato et al. 2001, 2004; Croton et al. 2006; Hopkins et al. 2006; Menci et al. 2008), the accretion activity in the universe has to be studied up to high redshifts and low luminosities. This requires large samples of active galactic nuclei (AGNs) spanning wide ranges of properties.

Although sizable samples have been collected in the optical and a decline in the quasar density between redshifts $\sim 2.5$ and 6 has been observed (Fan et al. 2001; Willott et al. 2003; Richards et al. 2006; Jiang et al. 2006), this evolution has been traced only by the most luminous $\left(-27.5<M_{1450}<-25.5\right)$ sources at redshift beyond 3. Only recently, Glikman et al. $(2010,2011)$ have given constraints on the faint end of the luminosity function in the optical band by using a sample of more than 40 faint $\left(M_{1450}<-22\right)$ quasars in the 3.74-5.06 redshift range. The presence of dust in high-redshift sources (Jiang et al. 2006; Gallerani et al. 2010) could, however, strongly affect their color selection and thus also the optical luminosity function.

Thanks to the sensitivity reached by the Chandra and XMMNewton satellites, sizable samples $(\sim 40$ sources in the largest one) of $z \sim 3-4 \mathrm{X}$-ray-detected AGNs have been collected (Silverman et al. 2005, 2008; Brusa et al. 2009; Yencho et al. 2009; Ebrero et al. 2009; Aird et al. 2010; Fiore 2010). $\mathrm{X}$-ray surveys are suitable for the selection of high-redshift lowluminosity AGNs because they are little affected by obscuration, unless Compton thick absorption (Brandt \& Hasinger 2005). Indeed, only a few Compton thick AGNs at $z>3$ have been recognized so far (Norman et al. 2002; Comastri et al. 2011; Gilli et al. 2011a). At the same time, X-ray surveys are limited in collecting large samples, either because wide area surveys have been not deep enough to detect $z>4$ AGNs or because deep surveys are too small and affected by cosmic variance. Only two $z>5$ spectroscopically confirmed quasars have been $\mathrm{X}$-ray selected so far, one in the Chandra Deep Field North (CDFN, $z=5.19$; Barger et al. 2005) and the other in the Chandra Large-Area Synoptic X-ray Survey $(z=5.4$; Steffen et al. 2004), both of them being unobscured type 1 quasars.

The space density of luminous quasars in the $X M M$ Cosmic Evolution Survey (COSMOS) at $3<z<4$ (Brusa et al. 2009) mimics the exponential decline observed in the optical. However, the higher redshift and lower luminosity regimes are still unexplored. The faint X-ray luminosity range $\left(\sim 10^{43} \mathrm{erg} \mathrm{s}^{-1}\right)$ 
Table 1

Summary of the $z>3$ Sample

\begin{tabular}{|c|c|c|c|c|c|c|c|c|c|c|c|c|}
\hline \multirow[t]{2}{*}{ Redshift } & \multicolumn{3}{|c|}{ Total } & \multicolumn{3}{|c|}{ Spec. } & \multicolumn{3}{|c|}{ Phot. } & \multicolumn{3}{|c|}{ Phot. $+1 \sigma>3$} \\
\hline & $S$ & $H$ & $F$ & $S$ & $H$ & $F$ & $S$ & $H$ & $F$ & $S$ & $H$ & $F$ \\
\hline$z>3$ & 81 & 14 & 6 & 29 & 2 & 0 & 36 & 10 & 4 & 16 & 2 & 2 \\
\hline$z>4$ & 14 & 1 & 1 & 6 & 0 & 0 & 7 & 1 & 0 & 1 & 0 & 1 \\
\hline$z>5$ & 4 & 0 & 1 & 2 & 0 & 0 & 2 & 0 & 0 & 0 & 0 & 1 \\
\hline \multirow[t]{2}{*}{ Redshift } & \multicolumn{3}{|c|}{ Total } & \multicolumn{3}{|c|}{ Spec. } & \multicolumn{3}{|c|}{ Phot. } & \multicolumn{3}{|c|}{ Phot. $+1 \sigma>3$} \\
\hline & $S$ & $H$ & $F$ & $S$ & $H$ & $F$ & $S$ & $H$ & $F$ & $S$ & $H$ & $F$ \\
\hline$z>3$ & 73 & 8 & 3 & 28 & 1 & 0 & 30 & 6 & 2 & 15 & 1 & 1 \\
\hline$z>4$ & 13 & 0 & 0 & 6 & 0 & 0 & 6 & 0 & 0 & 1 & 0 & 0 \\
\hline$z>5$ & 3 & 0 & 0 & 2 & 0 & 0 & 1 & 0 & 0 & 0 & 0 & 0 \\
\hline
\end{tabular}

Notes. Top: summary of the sources belonging to the high-redshift sample with secure spectroscopic, photometric redshift, and with $z_{\text {phot }}+\sigma_{\text {zphot }}>3$. Bottom: number of sources included in the space density computation, after applying a cut in flux limit.

is well sampled up to $z \sim 3$ (see, e.g., Hasinger 2008) but not many sources have been detected at this luminosity at higher redshifts.

The goal of this paper is to compute the space density of high-redshift AGNs in the $2-10 \mathrm{keV}$ rest-frame band that is little affected by obscuration (up to $N_{\mathrm{H}}=$ few $\times 10^{23} \mathrm{~cm}^{-2}$ ). For this reason, the main selection has been performed in the $0.5-2 \mathrm{keV}$ band, closely corresponding to the $2-10 \mathrm{keV}$ restframe band at $z>3$. In order to account for the presence of highly (up to $N_{\mathrm{H}} \sim 10^{24} \mathrm{~cm}^{-2}$ ) obscured AGNs, we also considered the sources with detection only in the observed $2-10$ or $0.5-10 \mathrm{keV}$ bands. Taking advantage of the mediumdepth, large-area Chandra survey of the COSMOS field (C-COSMOS; Elvis et al. 2009; Puccetti et al. 2009), we sample the $43.5 \mathrm{erg} \mathrm{s}^{-1}<\log L_{(2-10 \mathrm{keV})}<45 \mathrm{erg} \mathrm{s}^{-1}$ luminosity range to study the number counts and space density of high-redshift $(3<z<6.8)$ AGNs.

Throughout the paper we quote $\mathrm{AB}$ system magnitudes and we assume a cosmology with $H_{0}=70 \mathrm{~km} \mathrm{~s}^{-1} \mathrm{Mpc}^{-1}, \Omega_{M}=$ 0.3 , and $\Omega_{\Lambda}=0.7$.

\section{HIGH-REDSHIFT AGN SAMPLE}

The C-COSMOS survey (Elvis et al. 2009) covers the central $0.9 \mathrm{deg}^{2}$ of the COSMOS field to a depth of up to $200 \mathrm{ks}$ in the inner $0.5 \mathrm{deg}^{2}$. The C-COSMOS X-ray source catalog comprises 1761 point-like X-ray sources detected down to a maximum likelihood threshold detml $=10.8$ at least in one band $(0.5-2,2-8$, and $0.5-8 \mathrm{keV})$. This likelihood threshold corresponds to a probability of $\sim 5 \times 10^{-5}$ that a catalog source is instead a background fluctuation (Puccetti et al. 2009). Given the likelihood threshold above, the flux limit reached in the survey is $5.7 \times 10^{-16} \mathrm{erg} \mathrm{cm}^{-2} \mathrm{~s}^{-1}$ in the full band $(0.5-10 \mathrm{keV})$, $1.9 \times 10^{-16} \mathrm{erg} \mathrm{cm}^{-2} \mathrm{~s}^{-1}$ in the soft band $(0.5-2 \mathrm{keV})$, and $7.3 \times 10^{-16} \mathrm{erg} \mathrm{cm}^{-2} \mathrm{~s}^{-1}$ in the hard band $(2-10 \mathrm{keV})$.

The high-redshift AGN sample used in this work has been selected from the C-COSMOS X-ray catalog, combining the spectroscopic and photometric information available from the identification catalog of the $1761 \mathrm{X}$-ray C-COSMOS sources (F. Civano et al. 2011, in preparation). First, we selected all the sources with secure spectroscopic redshift from the identification catalog (870 sources). The brightest C-COSMOS sources, quite often associated with $X M M$-COSMOS sources, have been observed as compulsory targets by the zCOSMOS bright (Very Large Telescope (VLT)/VIMOS; Lilly et al. 2007, 2009) and the Magellan/IMACS (Trump et al. 2007, 2009) surveys limited to $i<22.5$. Recently, the C-COSMOS sources have been the primary targets of much deeper observations with the Keck/DEIMOS (to $i<25^{17}$ ) and VLT/VIMOS (zCOSMOS deep, to $B<25$ ) surveys. In this spectroscopic sample, 29 sources with soft band X-ray detection have redshift larger than 3, of which 6 are at $z>4$ and 2 at $z>5$. Only two sources with $z_{\text {spec }}>3$ do not have soft band detection, but only hard band detection. The highest redshift source with an optical spectrum, at $z_{\text {spec }}=5.3$, is one of the three spectroscopically confirmed members of the high- $z$ protocluster discovered in the COSMOS field, and its optical spectrum is reported in Capak et al. (2011). This quasar is the only X-ray-detected object in the protocluster.

In summary, the spectroscopic sample includes 31 sources.

Second, given that only $\sim 50 \%$ of the C-COSMOS sources have a spectroscopic redshift, the sources with photometric redshifts (Salvato et al. 2009; M. Salvato et al. 2011, in press), which are typically fainter $\left(i_{\mathrm{AB}}=21.5\right.$ and 23.8 are the mean optical magnitudes of the spectroscopic and photometric samples, respectively), need to be included, as these extend the sample to low luminosities and high redshifts. Given the large number of photometric bands (31, of which 12 are intermediate bands, suitable for the selection of emission lines), the spectral energy distribution (SED) of COSMOS sources can be used as a low-resolution spectrum. The COSMOS photometric redshifts for X-ray-selected sources have an accuracy of $\sigma_{\Delta z /\left(1+z_{\text {spec }}\right)}=$ 0.015 with a low number of outliers $(<6 \%)$, considering the sample as a whole. These numbers depend on the magnitude of the sources, but at $z>2.5$ a remarkably good accuracy of $\sigma_{\Delta z /\left(1+z_{\text {spec }}\right)}=0.011$ is achieved. The SEDs of the sources with photometric redshift larger than 3 have been visually inspected together with the photometric fitting and the probability distribution of all the possible solutions.

This process adds 36 sources with photometric redshift larger than 3 and a soft band detection ( 7 are at $z>4$ and 2 at $z>5$ ), plus 10 sources with hard and full band detection and 4 sources with a full band detection only. Adding the sources with photometric redshifts, we more than double the spectroscopic sample at each redshift (Table 1). The source with the highest photometric redshift is CID-2550 (soft-banddetected only), for which a photometric redshift of $z_{\text {phot }}=$ 6.8 has been computed (see M. Salvato et al. 2011, in press). To account for the broad and multiply peaked shape of the 17 The Keck survey is the result of a multi-year observing campaign (PIs:
Capak, Kartaltepe, Salvato, Sanders, Scoville). 
photometric redshift probability distribution $(P(z))$ and thus the possible contamination due to low-redshift sources, we computed the fraction of the $P(z)$ at $z_{\text {phot }}>3$ for each source with a photometric redshift only, to weight their contribution to the number counts and space density computation. Seventythree percent of these sources have a $P\left(z_{\text {phot }}>3\right)$ larger than 0.5 .

From the C-COSMOS identification catalog we also selected those sources detected in the soft, hard, or full band having a broad $P(z)$, such that $z_{\text {phot }}+\sigma_{\text {zphot }}>3$ and $z_{\text {phot }}<3$. Their SEDs have been visually inspected to check for possible problems, such as contaminated photometry due to nearby objects. A total of 20 sources ( 16 detected in the soft band, 2 in the hard and full band, and 2 in the full band only) were added to the main sample. We adopted $z=z_{\text {phot }}+\sigma_{\text {zphot }}$, and weighted their contribution to the number counts and to the space density by the fraction of the $P(z)$ at redshift $>3$. Only $15 \%$ of these sources have a $P\left(z_{\text {phot }}>3\right)$ larger than 0.5 .

Of the 81 soft-band-detected high- $z$ sources, 32 are also $X M M$-COSMOS sources (Brusa et al. 2010). Half were included in the Brusa et al. (2009) paper on $z>3$ sources, while five sources were below the XMM-COSMOS soft band threshold adopted for that study $\left(10^{-15} \mathrm{erg} \mathrm{cm}^{-2} \mathrm{~s}^{-1}\right)$. For the remaining 11 sources either a new version of the photometric redshift catalog (M. Salvato et al. 2011, in press), which employs a new and improved SED library and the addition of deep $H$-band photometry, suggests a higher $z$ solution (7 sources), or a newly available spectroscopic redshift (lower than $z<3$ ) superseded the previously available photometric one (4 sources). Brusa et al. (2009) also did not include the hard-band-detected sources. The new photometric redshifts (M. Salvato et al. 2011, in press) have been tuned using the C-COSMOS sources which, being fainter than the $X M M$-COSMOS ones, are typically more galaxy-dominated and thus in a few cases a different photometric redshift has been proposed with respect to Brusa et al. (2009, 2010).

In summary, the total $z>3 \mathrm{C}$-COSMOS sample includes 81 sources with $z>3$ (spectroscopic or photometric) plus 20 sources that are candidates to be at $z>3$ from their broad $P(z)$. The effective size of the sample, computed by summing the fraction of the $P(z)$ at $z>3$ for the 101 sources $(P(z)=1$ for the spectroscopic redshifts), is 73 sources. In the total sample, 81 sources are soft band selected, 14 are hard band selected, and 6 are full band selected (Table 1). The X-ray fluxes together with the redshifts of the sources are listed in Table 3.

Therefore, the C-COSMOS high-redshift sample increases the Brusa et al. (2009) sample by a factor of two. This is the largest available sample of $z>3$ X-ray-selected AGNs in a contiguous sky area, even when its effective size is considered. For comparison, in the 2Ms Chandra Deep Field South (CDFS) optical identification catalog (Luo et al. 2010; see also Silverman et al. 2010) there are 45 sources at $z>3$ (6 with spectroscopic and 39 photometric redshifts), of which 3 are at $z>4$, though none of these are spectroscopically identified. Considering the 4Ms CDFS catalog (Xue et al. 2011), 20 sources are added to the $z>3$ catalog, though with only photometric redshifts. F. Fiore et al. (2011, in press) will report the combination of the results obtained in all the above X-ray surveys.

\subsection{Optically Unidentified Sources}

There are also $18 \mathrm{C}$-COSMOS sources without a counterpart in the optical bands, but with a $K$ band and IRAC (10), only IRAC (6), or no detection (2). The optical images of these sources have been visually inspected to verify that their absence in the main optical catalog is not due to a source detection problem, and no optical emission has been found at the X-ray positions. Given the small number of bands in which these objects are detected, no photometric redshift is available for them. In $\mathrm{X}$-ray-selected samples, non-detection in the optical band has been often assumed to be a proxy for high redshift (e.g., Koekemoer et al. 2004), or for high obscuration, or a combination of both.

Four of the, eighteen, sources have no detection in the soft band suggesting high obscuration, possibly combined with high redshift. We considered the 14 soft-band-detected sources to be at $z>3$ on the basis of their optical non-detection and we included them in the derivation of the upper boundary of the $\log N-\log S$ curve (Section 3 ). Their contribution to the space density will be discussed in Section 4 .

\subsection{X-Ray Properties}

To allow for a useful comparison of the space density with model predictions, obscuration has to be taken into account. The sources in our sample have a low number of detected counts (28 is the median value in the $0.5-7 \mathrm{keV}$ band). In this count regime, spectral fit results are not stable, in particular when more than one free parameter is added to the fitting. Even if the fit converges the uncertainties on the parameters are large. For these reasons, we used the hardness ratio, defined as $\mathrm{HR}=(H-S) /(H+S)$ (where $H$ and $S$ are the counts in the $2-7 \mathrm{keV}$ and $0.5-2 \mathrm{keV}$ bands, respectively), to give a rough estimate of the obscuration affecting the sources and to derive the intrinsic luminosity. The upper or lower limits on the HR for the sources with detection only in one band (14 only hard and 37 only soft) have been computed by converting the $3 \sigma$ flux upper limit, in the band in which these sources are not detected, into counts.

To estimate the column density, curves of constant $N_{\mathrm{H}}$ as a function of redshift have been derived for two spectral slope values, $\Gamma=1.4$ and $\Gamma=2$. The flat spectral slope has been chosen to be consistent with the assumptions adopted in producing the original X-ray catalog (Puccetti et al. 2009). The steeper value is more representative of the intrinsic value if the spectrum is not affected by obscuration (Nandra \& Pounds 1994).

In Figure 1 (top panel) the curves of $N_{\mathrm{H}}=10^{20}, 5 \times$ $10^{22} \mathrm{~cm}^{-2}$, and $5 \times 10^{23} \mathrm{~cm}^{-2}$ are reported for $\Gamma=1.4$ (dashed lines) and $\Gamma=2$ (solid lines). Though there is a group of sources with HR indicative of significant obscuration, the low number of counts, the large HR error, and the similarity in this redshift range of the curves with widely different $N_{\mathrm{H}}$ values for the same spectral slope do not allow an accurate estimate of the column density for each source to be made. We also note that using two different intrinsic slopes implies quite different obscuration.

We thus classified as absorbed those sources whose HR $-1 \sigma$ are above the value corresponding to $N_{\mathrm{H}}=5 \times 10^{22} \mathrm{~cm}^{-2}$ at their own redshift for each spectral index. This criterion gives 9 $\mathrm{X}$-ray obscured sources (detected in both soft and hard bands) for $\Gamma=1.4$, or 32 sources for $\Gamma=2$. Hereafter, we will use $\Gamma=$ 2 , which gives a better estimate of the sources intrinsic column density. The luminosity of the candidate obscured sources have been corrected by using a correction factor derived from XSPEC (Arnaud 1996) by simulating an absorbed power law with the $N_{\mathrm{H}}$ computed above and $\Gamma=2$.

The 37 soft band sources with no detection in the hard band (reported as downward arrows in the top panel of Figure 1) have a very high upper limit on the HR, due to the conservative flux upper limit computed by Puccetti et al. (2009). Their 


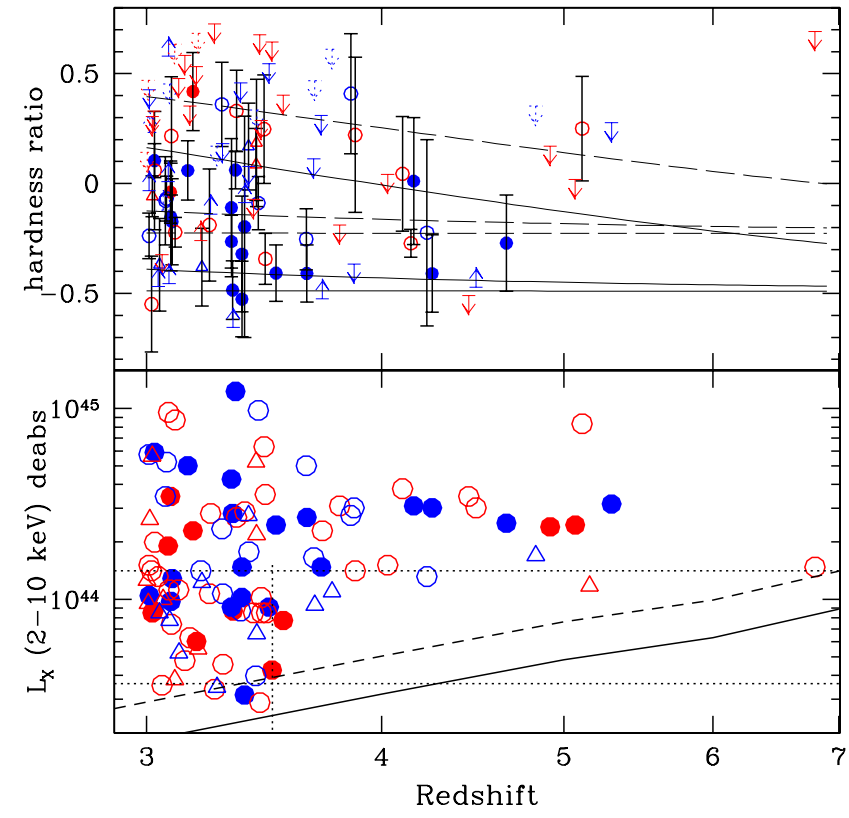

Figure 1. Top: hardness ratio vs. redshift. Blue $=$ type 1. Red $=$ not type 1 (see Section 2.3 for the definition). Filled = spectroscopic redshift. Open = photometric redshift. Sources with no hard band or soft band detection are reported as arrows. Three curves of constant $N_{\mathrm{H}}\left(10^{20}, 5 \times 10^{22} \mathrm{~cm}^{-2}\right.$, and $5 \times 10^{23} \mathrm{~cm}^{-2}$ ) are reported as dashed lines for $\Gamma=1.4$ and as solid lines for $\Gamma=2$. The sources with $z_{\text {phot }}+\sigma_{\text {zphot }}>3$ have been plotted as triangles or as dashed arrows if they do not have hard band or soft band detection. Bottom: the luminosity (computed with $\Gamma=1.4$ ) redshift plane for the objects in our sample. The continuous line represents the de-absorbed 2-10 keV luminosity limit of the survey computed from the $0.5-2 \mathrm{keV}$ limiting flux. The dashed line corresponds to the flux limit we imposed for the computation of the number counts and space density. Symbols are as in the top panel. The dotted black lines represent the luminosity limits used in the space density computation.

(A color version of this figure is available in the online journal.)

non-detection in the hard band is not necessarily due to obscuration but to the fact that these sources are very faint in the soft band and thus their hard band flux is below the flux limit of the survey.

For the 81 soft-band-detected objects, the $2-10 \mathrm{keV}$ restframe luminosity was computed by using the $0.5-2 \mathrm{keV}$ flux, which at $z>3$ corresponds to the hard $(>2 \mathrm{keV})$ rest-frame emission, assuming $\Gamma=2$. If needed, the absorption correction has been applied.

The $2-10 \mathrm{keV}$ rest-frame luminosity for the $14 z>3$ hard-band-only-detected sources (upward pointing arrows in Figure 1, top panel) has been computed by converting the observed 2-10 keV flux into rest-frame luminosity, using $\Gamma=2$. Although the non-detection in the soft band is suggestive of large column density $\left(>5 \times 10^{23} \mathrm{~cm}^{-2}\right)$, from the HR analysis only four of the HR lower limits lie above the $N_{\mathrm{H}}=5 \times 10^{22} \mathrm{~cm}^{-2}$ curve (for either choice of spectral slope assumptions), in the region of obscured sources.

For the four full-band-only-detected sources, the $2-10 \mathrm{keV}$ rest-frame luminosity has been computed by converting the full band flux and assuming the same spectral slopes.

The absorption-corrected hard X-ray luminosity (computed with $\Gamma=2$ ) versus redshift plane is reported in Figure 1 (bottom panel) together with the flux limit of the C-COSMOS survey (solid line).

\subsection{Optical Properties}

The $z>3$ C-COSMOS spectroscopic sub-sample (31 sources) includes 19 broad-line AGNs (type 1, FWHM >
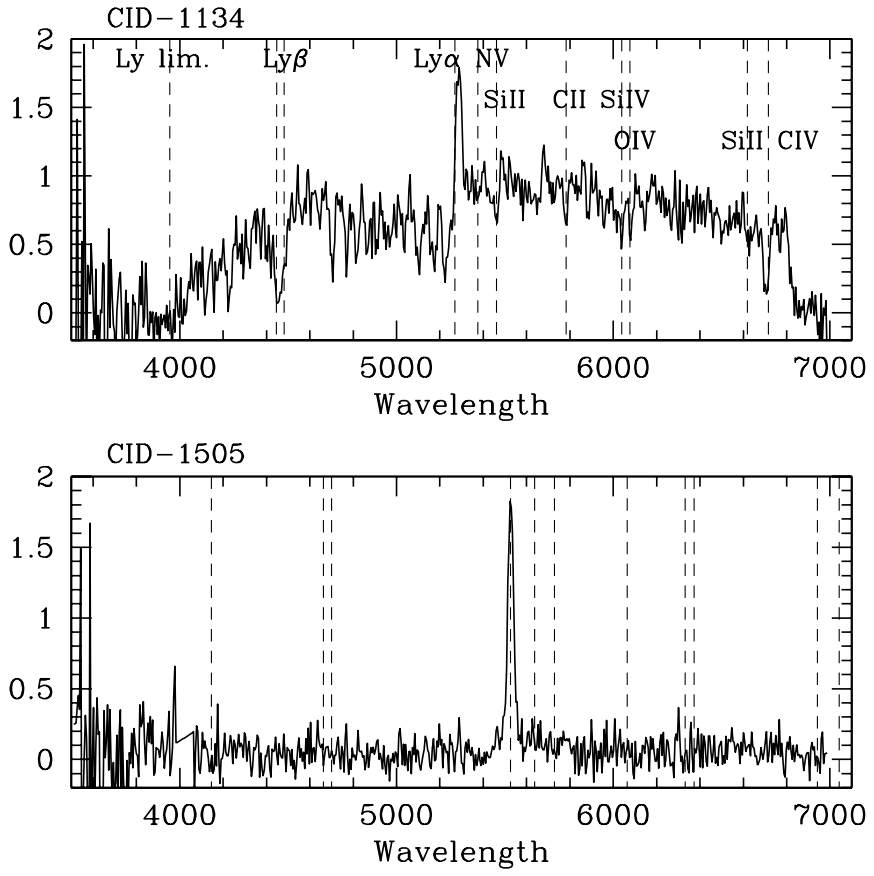

Figure 2. Optical spectra of sources CID-1134 (top, $z=3.335$ ) and CID1505 (bottom, $z=3.546$ ) from the VLT deep survey of the COSMOS field (zCOSMOS deep; Lilly et al. 2009). The spectra are plotted in arbitrary flux normalization. The dashed lines mark the same lines in both spectra (Ly limit, Ly $\beta, \mathrm{O}$ vI, Ly $\alpha, \mathrm{N}$ v, Si II, C II, Si IV and O IV, Si II and C IV).

$2000 \mathrm{~km} \mathrm{~s}^{-1}$ ) and 12 AGNs with narrow lines only (not type 1). The brightest $\left(i_{\mathrm{AB}} \sim 22-23\right)$ sources of the spectroscopic sample for which spectroscopic identification is available are type 1 AGNs. At fainter optical magnitudes $\left(i_{\mathrm{AB}}>23\right)$, we find an equal number of type 1 and not type 1 AGNs.

From the photometric fitting, an SED type can be derived for each source without spectroscopic classification. About half of the total sample is best fitted with an unobscured quasar template (type-1-like), and half with an obscured quasar template (not type-1-like; see Salvato et al. 2009 for more details on the SED templates).

The spectral types (solid circles) along with the SED fitting type (open circles) are reported in Figure 1 (type 1 in blue and not type 1 in red).

The optical spectra of X-ray-selected AGN show a broader range of properties than optical-selected samples. Even though a detailed analysis of the spectral properties (emission and absorption line intensity, continuum slope, extinction) will be the subject of a following paper, the variety of striking features seen in the spectra are briefly reported below.

The sources classified as type 1 AGNs have spectra with broad $\left(\mathrm{FWHM} \sim 5000 \mathrm{~km} \mathrm{~s}^{-1}\right)$ lines of either Ly $\alpha(1216 \AA)$ and C IV (1549 ̊), or C IV and [C III] (1909 ̊).

Among the narrow-line sources, three sources (Figure 2, top panel) show spectra typical of normal star-forming galaxies with a narrow Ly $\alpha$ emission and stellar absorption lines (Ly $\beta$, Si II at $1260 \AA, C$ II at $1334 \AA$, Si IV at $1393 \AA$, O IV at $1402 \AA$, and C IV at $1549 \AA$; Shapley et al. 2003). Interestingly, there is no hint of $\mathrm{C}$ IV line in emission, a typical signature for nuclear activity, in these objects. No spectra of this kind were found in the brighter $X M M$-COSMOS sample of $z>3$ AGNs (Brusa et al. 2009), while a few similar spectra have been found in the same redshift range in the ECDFS survey (Silverman et al. 2010), though with a lower signal-to-noise ratio. In particular, the source shown in 
Figure 2 (top panel) is not detected in the soft band but only in the hard band, suggesting the presence of high obscuration, consistent with the absence of nuclear emission lines.

Four sources show strong narrow $\left(\right.$ FWHM $\sim 1000 \mathrm{~km} \mathrm{~s}^{-1}$ ) emission lines (mostly Ly $\alpha$ ) over a faint, almost zero, continuum as shown in Figure 2 (bottom panel). The strength of their Ly $\alpha$ $\left(\mathrm{EW}_{\text {rest }} \sim 250 \AA\right)$ is comparable with the strongest lines found in Ly $\alpha$ emitter samples (see, e.g., Murayama et al. 2007; Cassata et al. 2011, and references therein). One of the sources, in this class, is the highest redshift narrow-line source $\left(z_{\mathrm{spec}}=5.07 ; \mathrm{H}\right.$. Ikeda et al. 2011, in press) in the sample. Without X-ray data, objects like those shown in Figure 2 would have been easily missed by optically based AGN surveys. The optical spectra themselves would not have allowed their classification as AGNs.

Broad absorption lines are visible in the spectra of a couple of sources, typically in the C IV doublet at $1549 \AA$. These indicate the presence of outflows, which can provide an important means of carrying material and energy out of the central region, and could thus be important for the studies of the feedback and for the enrichment of the intergalactic medium at high redshift.

\section{NUMBER COUNTS OF $z>3$ AGNs}

We derived the soft band $\log N-\log S$ of the $z>3$ and $z>4$ C-COSMOS samples by folding the observed flux distribution through the sky coverage area versus flux curve of the C-COSMOS survey (Puccetti et al. 2009).

In order to minimize the error associated with the most uncertain part of the sensitivity curve, we truncate the sample at the flux corresponding to $10 \%$ of the total area (dashed line in Figure 1). All the sources with a $0.5-2 \mathrm{keV}$ flux above $3 \times$ $10^{-16} \mathrm{erg} \mathrm{cm}^{-2} \mathrm{~s}^{-1}$ have been considered (73 objects out of 81 ; Column 1 of the lower Table 1).

The flux limit applied to the sample is consistent with the signal-to-noise ratio thresholds chosen by Puccetti et al. (2009), on the basis of extensive simulations, to avoid the Eddington bias in the computation of the number counts of the entire C-COSMOS sample. Thus, by applying a flux limit cut, we also reduce the Eddington bias affecting our sample.

The binned $\log N-\log S$ relations for two redshift ranges $(z>3$ and $z>4)$ are plotted in Figure 3 (red points, with associated Poissonian errors), together with the XMM-COSMOS number counts (Brusa et al. 2009, green points), estimated by using the most recent XMM-COSMOS identification catalog (Brusa et al. 2010).

The yellow shaded area represents an estimate of the maximum and minimum number counts relation at $z>3$ obtained by considering two different effects. First, we computed the $1 \sigma$ uncertainty in the sky coverage area for each source using the sky coverage as a function of flux of the C-COSMOS survey (see Figure 3 in Elvis et al. 2009) and the $1 \sigma$ uncertainty in the flux (computed as in Puccetti et al. 2009). This uncertainty is particularly important for sources with faint fluxes, where the error on the flux is large and the sky coverage curve is steep. Second, we considered the 14 sources with no-optical detection (seen in the soft band).

To compute the upper boundary of the shaded area, we included all the sources in the main sample (73 objects) plus the sources with no optical detection at their flux $+1 \sigma$ error. For the lower boundary, we used the flux $-1 \sigma$ error for all the sources in the main sample.

The blue and green solid curves $(z>3$ upper and $z>4$ lower) in Figure 3 correspond to the predictions of two different phenomenological models. The

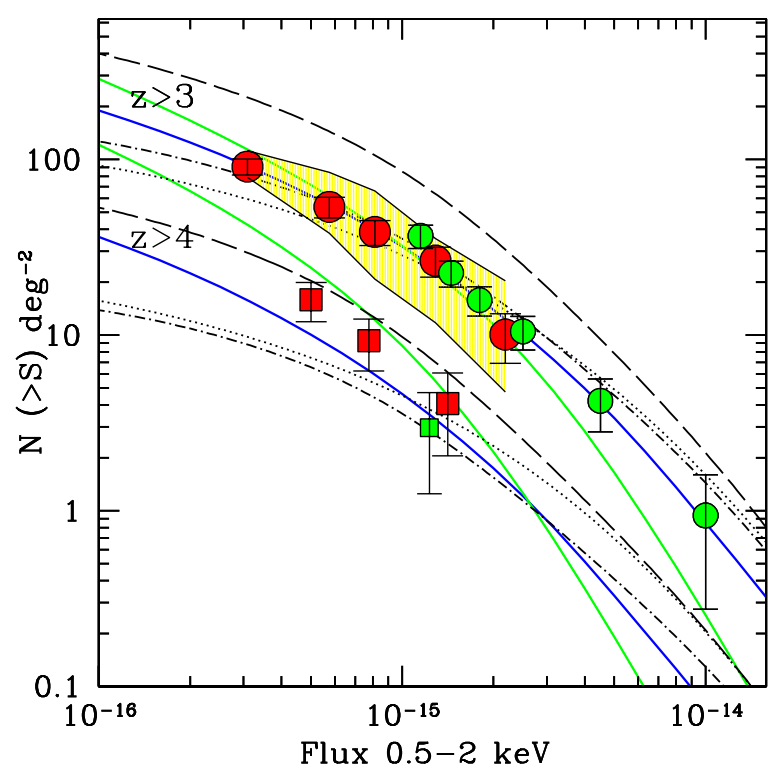

Figure 3. Binned $\log N-\log S$ relation (with associated Poissonian errors) of the $z>3$ (red circles) and $z>4$ (red squares) QSO population. The yellow shaded area represents the maximum and minimum number counts under the assumptions described in Section 3. The blue and green curves correspond to the prediction based on the Gilli et al. (2007) and the Aird et al. (2010, steeper curves) models, respectively. The green symbol represents the number counts for $z>3$ (circles) and $z>4$ (squares) QSOs estimated as in Brusa et al. (2009) for the XMM-COSMOS survey by using the most recent identification catalog (Brusa et al. 2010). The black dashed, dotted, and dot-dashed lines represent different predictions of a basic model of quasar activation by major mergers of dark matter halos (see description in Section 3).

(A color version of this figure is available in the online journal.)

first (flatter solid blue curve) is the X-ray background synthesis model of Gilli et al. (2007), ${ }^{18}$ based on the X-ray luminosity function observed at low redshift (e.g., Hasinger et al. 2005), parameterized with a luminosity dependent density evolution (LDDE), and a high-redshift exponential decline with the same functional form adopted by Schmidt et al. $\left(1995 ; \Phi(z)=\Phi\left(z_{0}\right) \times 10^{-0.43\left(z-z_{0}\right)}\right.$ and $\left.z_{0}=2.7\right)$ to fit the optical luminosity function between $z \sim 2.5$ and $z \sim 6$ (Fan et al. 2001 ), corresponding to one $e$-folding per unit redshift. The second one (steeper green solid curve) is the luminosity and density evolution model (LADE; Aird et al. 2010) which fits the hard X-ray luminosity function derived by Aird et al. (2010) using the 2Ms Chandra Deep Fields and the AEGIS-X (200 ks) survey to probe the faint end $\left(\log L_{X}<43 \mathrm{erg} \mathrm{s}^{-1}\right)$ and the high- $z$ $(z \sim 3)$ range.

At $z>3$, the C-COSMOS points confirm and tighten the agreement with the model predictions, previously found by Brusa et al. (2009), extending this agreement to fainter fluxes. At redshift $z>4$, where the XMM-COSMOS sample had only four sources, the C-COSMOS sample is three to four times larger, making it possible to compare the slope of the counts with models, which agree.

While at $z>3$ the two model predictions are very close, at $z>4$, where the models have different slopes, the errors on the data do not allow a firm preference of one of the two models.

While the above models are purely phenomenological, it is also possible to compare with physical models of quasar evolution. In Figure 3, we compare the number counts with

\footnotetext{
18 This model has been computed by using the POrtable Multi Purpose Application (POMPA) for AGN counts available at the Web site http://www.bo.astro.it/ gilli/counts.html.
} 

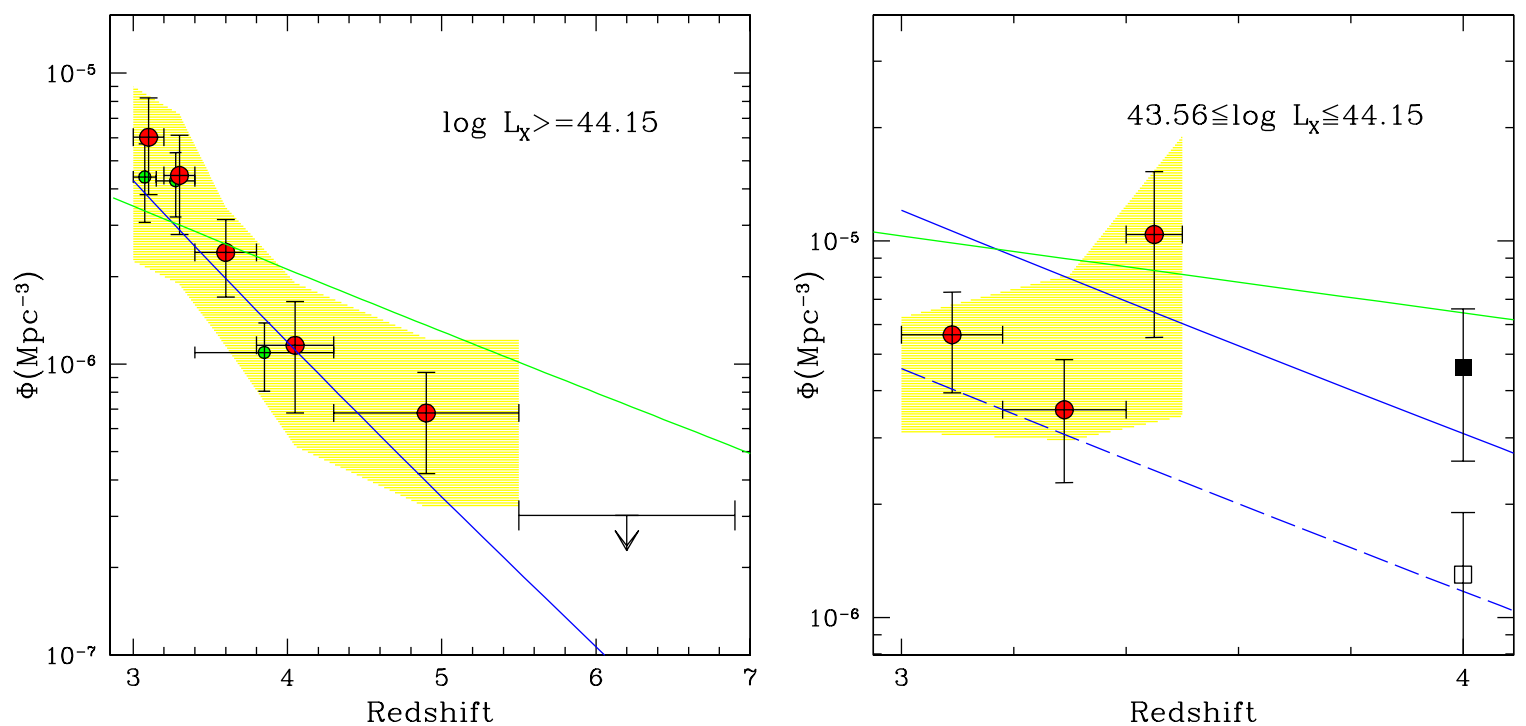

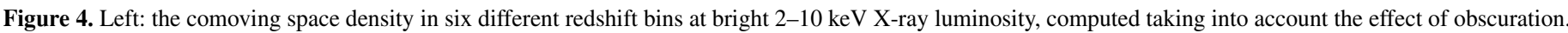

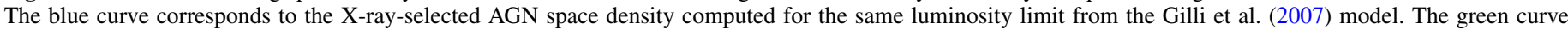

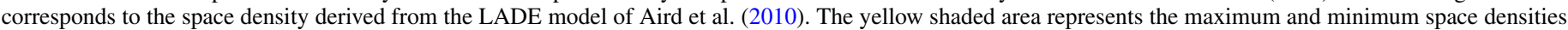

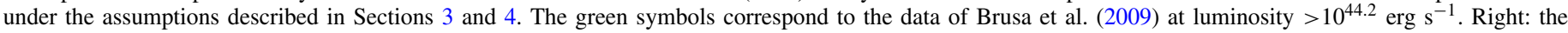

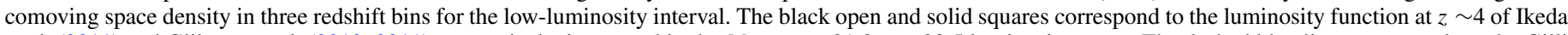

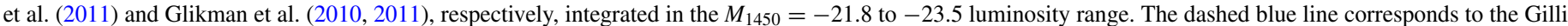
et al. (2007) model if only unobscured sources are considered.

(A color version of this figure is available in the online journal.)

the prediction of a basic model of quasar activation by major mergers of dark matter halos. Briefly, the model consists of a dark matter halo merger rate compatible with cosmological simulations and a quasar light curve that depends on the mass of the host and which describes the evolution of individual quasars (e.g., Wyithe \& Loeb 2003; Lapi et al. 2006; Shen 2009; Shankar 2009, 2010; Shankar et al. 2010). The initial mass of the BH at triggering is assumed to be a fixed fraction of its mass at the peak of activity. BH growth is regulated by a condition between the peak luminosity and the mass of the host halo at the triggering time. In Figure 3, we report the number counts obtained for each redshift range for a model with the same parameters as in Shen (2009; black dashed line) with minimum halo mass of $\sim 4 \times$ $10^{11} M_{\odot}$ (black dashed line). The dot-dashed line refers to a model with larger minimum host halo mass $\left(\sim 3 \times 10^{12} M_{\odot}\right.$, dot-dashed line) presented in a preliminary work by Shankar (2010). In both models, a prolonged quasar light curve (evolving as $t^{-\alpha}$ with $\alpha=2.5-3$ ), characterized by a long sub-Eddington post-peak phase, has been assumed. The curve from a model characterized by a lower minimum host halo masses $\left(10^{12} M_{\odot}\right)$ and negligible post-peak phase (dotted line; Shankar 2010 for details) is reported too. The discussion of this comparison is reported in Section 5.

\section{2-10 keV COMOVING SPACE DENSITY}

In order to avoid the Eddington bias at the faintest fluxes, we also applied a cut at the flux corresponding to $10 \%$ of the total area to the hard and full band flux. The fluxes associated with this area are $2 \times 10^{-15} \mathrm{erg} \mathrm{cm}^{-2} \mathrm{~s}^{-1}$ in the hard band and $1.2 \times$ $10^{-15} \mathrm{erg} \mathrm{cm}^{-2} \mathrm{~s}^{-1}$ in the full band. The number of sources used in the derivation of the space density is reported in Table 1 (bottom) in each band.

Including soft-, hard-, and full-band-detected sources allow us to compute a space density which takes into account both unobscured, emitting more at softer energies, and obscured sources, emitting at more at harder energies, as shown in Section 2.2, without having to introduce any further correction or assumption.

The comoving space densities were computed with the $1 / V_{\max }$ method (Schmidt 1968), which takes into account the fact that more luminous objects are detectable over a larger volume. The statistical uncertainty has been computed following Marshall et al. (1983). We also used the method proposed by Avni \& Bahcall (1980) to account for the fact that each object could have been found in any point of the survey, and thus at a different X-ray depth. The maximum available volume, over which each source can be detected, has been computed by using the formula

$$
V_{\max }=\int_{z_{\min }}^{z_{\max }} \Omega\left(f\left(L_{X}, z, N_{\mathrm{H}}\right)\right) \frac{d V}{d z} d z,
$$

where $\Omega\left(f\left(L_{X}, z, N_{\mathrm{H}}\right)\right)$ is the sky coverage at the flux $f\left(L_{X}, z\right)$ corresponding to a source with absorption column density $N_{\mathrm{H}}$ and unabsorbed luminosity $L_{X}$, and $z_{\max }$ is the maximum redshift at which the source can be observed at the flux limit of the survey. If $z_{\max }>z_{\text {up,bin }}$, then $z_{\max }=z_{\text {up,bin }}$, where $z_{\text {up,bin }}$ is the upper boundary of the bin. More specifically, for unabsorbed sources we adopted the observed rest-frame 2-10 keV luminosity, while for obscured ones the unabsorbed luminosity was derived assuming the best-fit column density as obtained from the HR (as explained in Section 2.2). We computed the space density using the luminosities derived with $\Gamma=2$. The contribution of sources with photometric redshift to the space density is weighted for the fraction of $P(z)$ at $z>3$ (see Section 2).

The comoving space density is shown in Figure 4. In order to match the flux limit imposed above (see Figure 1, dashed line) and to have a complete sample over a given redshift range, we divided the sample into two luminosity intervals. At high luminosity (left panel), we computed the space density in six redshift bins $(z=3-6.2)$ at $\log L_{X}>44.15 \mathrm{erg} \mathrm{s}^{-1}$, 
Table 2

Summary of the Sources in Each Luminosity and Redshift Bin

\begin{tabular}{|c|c|c|c|c|}
\hline \multirow[t]{2}{*}{$z$ Bin } & \multicolumn{4}{|c|}{$\begin{array}{c}\text { Number of Sources } \\
\left(\log L_{(2-10 \mathrm{keV})}>44.15 \mathrm{erg} \mathrm{s}^{-1}\right)\end{array}$} \\
\hline & $N$ & $N(\text { Type })^{\mathrm{a}}$ & $N_{\text {lower }} \mathrm{b}$ & $N_{\text {upper }}{ }^{\mathrm{b}}$ \\
\hline 3.1 & 15 & $5-10$ & 10 & 21 \\
\hline 3.3 & 10 & $7-3$ & 6 & 13 \\
\hline 3.6 & 12 & $6-6$ & 8 & 13 \\
\hline 4.05 & 6 & $3-3$ & 5 & 8 \\
\hline 4.9 & 7 & $3-4$ & 5 & 9 \\
\hline 6.2 & 0 & 0 & 0 & 1 \\
\hline \multirow[t]{2}{*}{$z$ Bin } & \multicolumn{4}{|c|}{ Number of Sources } \\
\hline & $N$ & $N(\text { Type })^{\mathrm{a}}$ & $N_{\text {lower }}{ }^{\mathrm{b}}$ & $N_{\text {upper }}{ }^{\mathrm{b}}$ \\
\hline 3.09 & 15 & $5-10$ & 12 & 13 \\
\hline 3.29 & 8 & $5-3$ & 9 & 11 \\
\hline 3.45 & 7 & $3-4$ & 6 & 9 \\
\hline
\end{tabular}

Notes.

a The number of sources per bin per optical type: first the number of type 1 sources and second the number of not type 1 sources.

$\mathrm{b}$ The number of sources per bin included in the lower and upper boundaries is reported.

while for the low-luminosity sample (right panel) a cut at $\log L_{X}=43.56 \mathrm{erg} \mathrm{s}^{-1}$ and $z=3.5$ (dotted lines in Figure 1) has been imposed. In Table 2, the number of sources in each redshift bin for the two luminosity ranges is reported.

We also estimated space density upper and lower boundaries by taking into account the X-ray flux errors. If a source has been excluded from the main sample because its flux is lower than the flux limit applied, the same source could be included in the upper boundary sample if its flux $+1 \sigma$ error exceeds the flux limit. Likewise, if a source has been included in the main sample because its flux is higher than the flux limit applied, the same source could be excluded by the lower boundary sample if its flux $-1 \sigma$ error is lower than the flux limit. For example, the source with photometric redshift $z=6.8$ (CID-2550) has a soft band flux below the chosen flux limit, but the flux $+1 \sigma$ error exceeds this limit so it is included in the upper boundary and, being the only source in that redshift bin, it has been plotted as an upper limit (at $3 \sigma$ ).

The yellow shaded area includes the above uncertainties affecting the computation of the space density, i.e., the flux errors and thus errors on the maximum volume associated with each source. In Columns 3 and 4 of Table 2, the number of sources when the flux errors are considered is reported.

As explained in Section 2.3, the 18 sources with no optical band detection have not been included in the space density boundaries. However, we computed the space density with the assumption that all 18 sources were at the redshift corresponding to the first bin, then to the second bin, and so on. The space density values computed in this case, in the first three bins, are within the yellow shaded area. If all the non-optically identified sources are at $z=4.05$ or at $z=4.9$, respectively, the number of sources per bin will be four times higher than the value reported in Table 2, and the same would be true for the space density. However, this last unlikely option is the most extreme and we have not included this correction in the derivation of the upper boundary in these two high-redshift bins.

The space density in the two luminosity ranges is compared with the predictions, at the same luminosity threshold, from the same Gilli et al. (2007) model used for the $\log N-\log S$ (blue solid line), including in the model all the sources up to a column density of $10^{24} \mathrm{~cm}^{-2}$. We also compare it with the LADE model (Aird et al. 2010; green solid line).

At $z \sim 3$, as already found for the number counts, the Gilli et al. (2007) and Aird et al. (2010) declining space density provides a good representation of the observed data. At $z>4$, the LADE model overpredicts the observed data in the highluminosity range, but, when the uncertainties are taken into account (shaded yellow area), the data lie between the two models.

No X-ray space density for these redshifts has been reported previously at low luminosities (43.56 erg s $\mathrm{e}^{-1}<\log L_{X}<$ $44.15 \mathrm{erg} \mathrm{s}^{-1}$; Figure 4 , right panel). The size of the sample (32 sources), however, does not allow us to discriminate between models.

In order to compare our data with other recent observations, we derived the space density at similar luminosity from the optical (at $1450 \AA$ ) luminosity functions of broad-line quasars at $z \sim 4$ of Glikman et al. (2010, 2011) and Ikeda et al. (2011). For both authors, we used their two power-law luminosity functions, since we are probing the region around $M_{\star}$ where strong curvature is present and both slopes contribute to the shape. Assuming a relation between the X-ray luminosity at $2 \mathrm{keV}$ and the luminosity at $1500 \AA\left(\alpha_{o x}=1.929-0.119 \log L_{1500 \AA}\right.$; Young et al. 2010), we converted the $43.56 \mathrm{erg} \mathrm{s}^{-1}<\log L_{X}<$ $44.15 \mathrm{erg} \mathrm{s}^{-1}$ range (in the rest-frame hard band) into absolute magnitude at $1500 \AA$. We then integrated their luminosity functions between $M_{1450}=-21.8$ and -23.5 to obtain the corresponding space density at $z \sim 4$. The two values are reported in Figure 4 (right panel; Glikman et al. 2011 as a filled square; Ikeda et al. 2011 as an open square).

The space densities derived from the two studies differ by a factor of three (as reported in Ikeda et al. 2011), and the reason for this difference is not well understood. It should be noted that both estimates are based on rather small samples (40 sources in Glikman et al. 2011 and 8 sources in Ikeda et al. 2011) and thus large errors should be taken into account. Although the Glikman et al. (2011) result is consistent with the predictions of the Gilli et al. (2007) model when the obscured sources are taken into account (blue solid line), as well as the Aird et al. (2010) model, while the space density derived from the Ikeda et al. (2011) is in agreement with the prediction when only unobscured sources are considered (dashed line; Gilli et al. 2007), it is not clear how to explain the difference given that both samples include only broad-line quasars. However, if we consider the optical type for our sources in each bin, we also do not find a good agreement with the models: in the first bin the majority of sources are not type 1 (see Table 2, Column 2), but the space density value is closer to the prediction when only unobscured sources are considered (dashed line).

\section{DISCUSSION AND CONCLUSION}

In this paper we have selected a sample of 81 high-redshift $(z>3)$ sources plus 20 candidate high-redshift sources in the C-COSMOS survey to study the evolution and space density of high-redshift AGNs in both the high- and low-luminosity regimes. This sample is the largest available sample of $z>3$ $X$-ray-selected AGNs in a contiguous sky area. Using the photometric redshifts and their associated $P(z)$, we also were able to compute the effective size of the sample (73 sources), by summing the probability of being at $z>3$ of all the sources. 
Table 3

Properties of $z>3$ AGNs from the C-COSMOS Sample

\begin{tabular}{|c|c|c|c|c|c|c|c|c|c|c|c|c|}
\hline Chid $^{\mathrm{a}}$ & IAU Name ${ }^{\mathrm{b}}$ & $F_{0.5-2 \mathrm{keV}^{\mathrm{c}}}$ & Error & $F_{2-10 \mathrm{keV}^{\mathrm{c}}}$ & Error & $F_{0.5-10 \mathrm{keV}^{\mathrm{c}}}$ & Error & $z^{\mathrm{d}}$ & $z_{\text {spec }}{ }^{\mathrm{d}}$ & $z_{\text {phot }}{ }^{\mathrm{d}}$ & flag $^{\mathrm{e}}$ & $\mathrm{wg}^{\mathrm{f}}$ \\
\hline 64 & CXOCJ100127.5+020837 & $2.37 \mathrm{e}-15$ & $3.00 \mathrm{e}-16$ & $6.24 \mathrm{e}-15$ & $1.41 \mathrm{e}-15$ & $9.03 e-15$ & $1.02 \mathrm{e}-15$ & 3.328 & 3.328 & 3.359 & 1 & 1.00 \\
\hline 83 & CXOCJ100051.4+022830 & $1.93 \mathrm{e}-15$ & $2.58 \mathrm{e}-16$ & $7.67 \mathrm{e}-15$ & $1.20 \mathrm{e}-15$ & $8.90 \mathrm{e}-15$ & $8.94 \mathrm{e}-16$ & 3.074 & & 3.074 & 1 & 0.99 \\
\hline 113 & CXOCJ100050.1+022855 & $2.29 \mathrm{e}-15$ & $3.20 \mathrm{e}-16$ & $3.67 \mathrm{e}-15$ & $1.17 \mathrm{e}-15$ & $7.75 e-15$ & $9.91 \mathrm{e}-16$ & 3.333 & 3.333 & 3.373 & 1 & 1.00 \\
\hline 124 & CXOCJ100049.2+023010 & $1.30 \mathrm{e}-15$ & $3.41 \mathrm{e}-16$ & $5.23 \mathrm{e}-15$ & $1.60 \mathrm{e}-15$ & $5.96 \mathrm{e}-15$ & $1.18 \mathrm{e}-15$ & 3.070 & $\ldots$ & 3.070 & 1 & 1.00 \\
\hline 270 & CXOCJ100025.7+014533 & $5.52 \mathrm{e}-16$ & $2.05 e-16$ & $2.63 e-15$ & $1.17 \mathrm{e}-15$ & $2.39 \mathrm{e}-15$ & $7.39 \mathrm{e}-16$ & 4.160 & 4.160 & 3.946 & 1 & 1.00 \\
\hline 293 & CXOCJ100049.1+014409 & $5.49 \mathrm{e}-16$ & $1.96 \mathrm{e}-16$ & $2.83 e-15$ & $1.08 \mathrm{e}-15$ & $3.08 \mathrm{e}-15$ & $7.48 \mathrm{e}-16$ & 4.104 & & 4.104 & 1 & 0.69 \\
\hline 308 & СXОCJ095856.6+021047 & $1.36 \mathrm{e}-15$ & $2.30 \mathrm{e}-16$ & $2.59 \mathrm{e}-15$ & $9.99 \mathrm{e}-16$ & $5.01 \mathrm{e}-15$ & $7.74 \mathrm{e}-16$ & 4.255 & 4.255 & 4.244 & 1 & 1.00 \\
\hline 317 & CXOCJ095912.8+020826 & $8.58 \mathrm{e}-16$ & $1.79 \mathrm{e}-16$ & $1.81 \mathrm{e}-15$ & $8.02 \mathrm{e}-16$ & $3.19 \mathrm{e}-15$ & $6.04 \mathrm{e}-16$ & 3.048 & $\ldots$ & 2.834 & -1 & 0.21 \\
\hline 349 & CXOCJ100001.0+020220 & $1.74 \mathrm{e}-15$ & $2.43 \mathrm{e}-16$ & $3.36 \mathrm{e}-15$ & $9.18 \mathrm{e}-16$ & $6.13 e-15$ & $7.61 \mathrm{e}-16$ & 3.515 & 3.515 & 3.506 & 1 & 1.00 \\
\hline 407 & CXОСJ095914.0+021849 & $2.40 \mathrm{e}-15$ & $3.18 \mathrm{e}-16$ & $5.39 \mathrm{e}-15$ & $1.23 \mathrm{e}-15$ & $8.74 \mathrm{e}-15$ & $1.00 \mathrm{e}-15$ & 3.469 & $\ldots$ & 3.469 & 1 & 0.79 \\
\hline 413 & CXOCJ095928.7+021738 & $2.32 \mathrm{e}-15$ & $2.80 \mathrm{e}-16$ & $1.21 \mathrm{e}-14$ & $1.46 \mathrm{e}-15$ & $1.21 \mathrm{e}-14$ & $1.02 \mathrm{e}-15$ & 3.345 & 3.345 & 3.397 & 1 & 1.00 \\
\hline 507 & CXOCJ095926.0+022433 & $4.91 \mathrm{e}-15$ & $3.95 \mathrm{e}-16$ & $1.30 \mathrm{e}-14$ & $1.46 \mathrm{e}-15$ & $1.88 \mathrm{e}-14$ & $1.23 \mathrm{e}-15$ & 4.145 & - & 4.145 & 1 & 1.00 \\
\hline 521 & CXOCJ095902.8+022605 & $2.21 \mathrm{e}-15$ & $3.83 \mathrm{e}-16$ & $6.13 e-15$ & $1.47 \mathrm{e}-15$ & $8.98 \mathrm{e}-15$ & $1.22 \mathrm{e}-15$ & 3.647 & . & 3.647 & 1 & 1.00 \\
\hline 529 & CXOCJ095955.5+021854 & $2.18 \mathrm{e}-15$ & $2.68 \mathrm{e}-16$ & $8.96 \mathrm{e}-15$ & $1.26 \mathrm{e}-15$ & $1.01 \mathrm{e}-14$ & $9.28 \mathrm{e}-16$ & 3.021 & $\ldots$ & 2.988 & -1 & 0.36 \\
\hline 558 & CXOCJ095931.7+023018 & $4.47 \mathrm{e}-15$ & $3.77 \mathrm{e}-16$ & $1.31 \mathrm{e}-14$ & $1.48 \mathrm{e}-15$ & $1.81 \mathrm{e}-14$ & $1.21 \mathrm{e}-15$ & 3.107 & $\ldots$ & 3.107 & 1 & 0.85 \\
\hline 666 & CXOCJ095913.8+014838 & $6.07 \mathrm{e}-16$ & $2.50 \mathrm{e}-16$ & $1.76 \mathrm{e}-15$ & $1.39 \mathrm{e}-15$ & $2.60 \mathrm{e}-15$ & $9.39 \mathrm{e}-16$ & 4.228 & $\ldots$ & 4.228 & 1 & 0.25 \\
\hline 673 & CXOCJ100047.8+014352 & $3.83 \mathrm{e}-16$ & $1.70 \mathrm{e}-16$ & $2.63 e-15$ & $1.06 \mathrm{e}-15$ & $2.18 \mathrm{e}-15$ & $6.62 \mathrm{e}-16$ & 3.432 & $\ldots$ & 2.809 & -1 & 0.46 \\
\hline 693 & CXOCJ095924.3+021635 & $1.16 \mathrm{e}-15$ & $2.06 e-16$ & $1.67 \mathrm{e}-15$ & $7.37 e-16$ & $4.13 e-15$ & $6.48 \mathrm{e}-16$ & 3.371 & 3.371 & 3.313 & 1 & 1.00 \\
\hline 700 & СXОCJ095924.3+022536 & $4.12 \mathrm{e}-16$ & $1.36 \mathrm{e}-16$ & $3.78 \mathrm{e}-15$ & $9.53 e-16$ & $2.81 \mathrm{e}-15$ & $5.76 \mathrm{e}-16$ & 3.349 & $\ldots$ & 3.349 & 1 & 0.91 \\
\hline 781 & CXOCJ100024.2+022510 & $9.09 \mathrm{e}-16$ & $1.90 \mathrm{e}-16$ & $2.35 e-15$ & $9.99 \mathrm{e}-16$ & $3.52 \mathrm{e}-15$ & $6.85 e-16$ & 4.660 & 4.660 & 4.541 & 1 & 1.00 \\
\hline 917 & CXOCJ100046.2+021311 & $6.41 \mathrm{e}-16$ & $1.66 \mathrm{e}-16$ & $2.01 \mathrm{e}-15$ & $8.63 e-16$ & $2.93 e-15$ & $6.27 \mathrm{e}-16$ & 3.090 & 3.090 & 3.076 & 1 & 1.00 \\
\hline 947 & CXOCJ100111.3+020855 & $5.05 \mathrm{e}-16$ & $1.59 \mathrm{e}$ & $1.81 \mathrm{e}$ & 9.96 & & 6.23 & & 3.328 & & 1 & 1.00 \\
\hline 953 & CXOCJ100050.5+022329 & $8.40 \mathrm{e}-16$ & $1.76 \mathrm{e}-16$ & $2.73 e-15$ & $9.44 \mathrm{e}-16$ & $3.49 \mathrm{e}-15$ & $6.43 e-16$ & 3.095 & 3.095 & 3.084 & 1 & 1.00 \\
\hline 1040 & CXOCJ100054.2+014759 & $3.74 \mathrm{e}-16$ & $1.34 \mathrm{e}-16$ & $3.69 \mathrm{e}-15$ & $9.59 \mathrm{e}-16$ & $3.02 \mathrm{e}-15$ & $5.93 e-16$ & 3.290 & $\ldots$ & 3.290 & 1 & 0.94 \\
\hline 1112 & CXOCJ100134.7+015837 & $3.17 \mathrm{e}-15$ & $4.03 \mathrm{e}-16$ & $9.00 \mathrm{e}-15$ & $1.60 \mathrm{e}-15$ & $1.24 \mathrm{e}-14$ & $1.28 \mathrm{e}-15$ & 3.009 & & 3.009 & 1 & 0.40 \\
\hline 1118 & CXOCJ095931.0+021332 & $1.74 \mathrm{e}-15$ & $2.38 \mathrm{e}-16$ & $3.33 e-15$ & $9.29 \mathrm{e}-16$ & -15 & 7.4 & 3.650 & 3.650 & 3.647 & 1 & 1.00 \\
\hline 1147 & CXOCJ100001.8+020857 & $4.96 \mathrm{e}-16$ & $1.44 \mathrm{e}-16$ & $3.16 \mathrm{e}-15$ & $9.03 e-16$ & $2.86 \mathrm{e}-15$ & $5.77 \mathrm{e}-16$ & 3.398 & & 2.849 & -1 & 0.45 \\
\hline 1197 & CXOCJ095934.6+022559 & $2.45 \mathrm{e}-16$ & $1.19 \mathrm{e}-16$ & $7.63 e-16$ & $7.09 \mathrm{e}-16$ & 16 & 4.42 & 3.382 & 3.382 & 3.391 & 1 & 1.00 \\
\hline 1304 & CXOCJ095926.5+022316 & $1.87 \mathrm{e}-16$ & $1.01 \mathrm{e}-16$ & $1.36 \mathrm{e}-15$ & $6.92 \mathrm{e}-16$ & $1.09 \mathrm{e}-15$ & $4.16 \mathrm{e}-16$ & 3.873 & & 3.873 & 1 & 0.51 \\
\hline 1656 & CXOCJ100105.1+013649 & $1.09 \mathrm{e}-15$ & $4.38 \mathrm{e}-16$ & $8.17 \mathrm{e}-15$ & $2.77 e-15$ & $5.87 \mathrm{e}-15$ & $1.69 \mathrm{e}-15$ & 3.464 & . & 3.464 & 1 & 1.00 \\
\hline 3391 & CXOCJ100010.2+015219 & $8.10 \mathrm{e}-16$ & $1.87 \mathrm{e}-16$ & $1.89 \mathrm{e}-15$ & $1.02 \mathrm{e}-15$ & $3.01 \mathrm{e}-15$ & $6.85 e-16$ & 3.371 & 3.371 & 3.332 & 1 & 1.00 \\
\hline 3397 & CXOCJ100014.9+014321 & $7.61 \mathrm{e}-16$ & $2.68 \mathrm{e}-16$ & $3.93 e-15$ & $1.60 \mathrm{e}-15$ & $4.58 \mathrm{e}-15$ & $1.09 \mathrm{e}-15$ & 3.030 & & 3.030 & 1 & 0.71 \\
\hline 43 & CXOCJ100043.4+020433 & $1.08 \mathrm{e}-15$ & $1.85 \mathrm{e}-16$ & $-1.27 \mathrm{e}-14$ & $\ldots$ & $4.21 \mathrm{e}-15$ & $6.11 \mathrm{e}-16$ & 3.010 & 3.010 & 3.013 & 1 & 1.00 \\
\hline 689 & CXOCJ100139.6+015603 & $1.07 \mathrm{e}-15$ & $2.88 \mathrm{e}-16$ & $-6.41 e-15$ & & $3.56 \mathrm{e}-15$ & $9.32 \mathrm{e}-16$ & & $\ldots$ & 3.681 & 1 & 0.39 \\
\hline 691 & CXOCJ095914.9+021658 & $8.90 \mathrm{e}-16$ & $1.86 \mathrm{e}-16$ & $-6.12 \mathrm{e}-15$ & $\ldots$ & 3.15 & 6.1 & & & 3.293 & 1 & 1.00 \\
\hline 720 & J095938.2+023711 & $5.72 \mathrm{e}-16$ & $2.17 \mathrm{e}-16$ & $-5.69 \mathrm{e}-15$ & $\ldots$ & $2.36 \mathrm{e}-15$ & 7.6 & & .. & 2.583 & -1 & 0.67 \\
\hline 815 & CXOCJ100002.2+015109 & $7.85 \mathrm{e}-16$ & $2.03 \mathrm{e}-16$ & $-4.04 \mathrm{e}-15$ & $\ldots$ & $2.83 e-15$ & $7.24 \mathrm{e}-16$ & 4.030 & $\ldots$ & 4.030 & 1 & 1.00 \\
\hline 890 & CXOCJ095940.7+022043 & $8.75 \mathrm{e}-16$ & $1.95 \mathrm{e}-16$ & $-7.81 \mathrm{e}-15$ & $\ldots$ & $2.64 \mathrm{e}-15$ & $6.39 \mathrm{e}-16$ & 3.021 & 3.021 & 3.042 & 1 & 1.00 \\
\hline 892 & CXOCJ100011.1+022202 & $3.61 \mathrm{e}-16$ & $1.23 \mathrm{e}-16$ & $-7.68 \mathrm{e}-15$ & $\ldots$ & $1.32 \mathrm{e}-15$ & $4.23 \mathrm{e}-16$ & 3.106 & & 2.326 & -1 & 0.36 \\
\hline 955 & CXOCJ100050.1+022618 & $9.17 \mathrm{e}-16$ & $1.88 \mathrm{e}-16$ & $-8.26 \mathrm{e}-15$ & $\ldots$ & 15 & $6.31 \mathrm{e}-16$ & 3.715 & 3.715 & 3.561 & 1 & 1.00 \\
\hline 1084 & CXOCJ095959.6+015828 & $1.30 \mathrm{e}-15$ & $2.20 \mathrm{e}-16$ & $-8.19 \mathrm{e}-15$ & $\ldots$ & $3.87 \mathrm{e}-15$ & $6.57 \mathrm{e}-16$ & 3.002 & $\ldots$ & 2.910 & -1 & 0.06 \\
\hline 1236 & CXOCJ095922.9+022853 & $6.92 \mathrm{e}-16$ & $2.21 \mathrm{e}-16$ & $-8.53 e-15$ & $\ldots$ & $2.25 \mathrm{e}-15$ & $7.57 \mathrm{e}-16$ & 3.366 & $\ldots$ & 3.366 & 1 & 1.00 \\
\hline 1392 & CXOCJ100149.1+015802 & $6.70 \mathrm{e}-16$ & $2.75 \mathrm{e}-16$ & $-1.07 \mathrm{e}-14$ & $\ldots$ & $3.71 \mathrm{e}-15$ & $1.15 \mathrm{e}-15$ & 3.485 & 3.485 & 3.490 & 1 & 1.00 \\
\hline 1505 & CXOCJ100023.2+020117 & $5.38 \mathrm{e}-16$ & $1.67 \mathrm{e}-16$ & $-5.96 \mathrm{e}-15$ & & $1.51 \mathrm{e}-15$ & $6.07 \mathrm{e}-16$ & 3.546 & 3.546 & 3.361 & 1 & 1.00 \\
\hline 1509 & CXOCJ100116.2+020017 & $6.51 \mathrm{e}-16$ & $1.91 \mathrm{e}-16$ & $-2.66 e-15$ & & $2.29 \mathrm{e}-15$ & $7.18 \mathrm{e}-16$ & 3.419 & $\ldots$ & 3.419 & 1 & 0.81 \\
\hline 1654 & CXOCJ100104.1+014203 & $1.36 \mathrm{e}-15$ & $3.75 \mathrm{e}-16$ & $-7.65 e-15$ & & $4.69 \mathrm{e}-15$ & $1.11 \mathrm{e}-15$ & & & 3.400 & 1 & 0.92 \\
\hline 1658 & CXOCJ100109.0+013902 & $1.70 \mathrm{e}-15$ & $4.65 \mathrm{e}-16$ & $-3.78 \mathrm{e}-15$ & $\cdots$ & $4.65 \mathrm{e}$ & $1.27 \mathrm{e}-15$ & 3. &.. & 3.867 & 1 & 0.79 \\
\hline 1660 & $1.9+014116$ & $7.48 \mathrm{e}-16$ & $2.95 \mathrm{e}-16$ & $-9.54 \mathrm{e}-15$ & $\ldots$ & $3.76 \mathrm{e}-15$ & & & $\ldots$ & 2.946 & -1 & 0.26 \\
\hline 1672 & CXOCJ100122.6+013809 & $1.83 e-15$ & $5.56 \mathrm{e}-16$ & $-5.93 e-15$ & $\ldots$ & $5.21 \mathrm{e}-15$ & $1.70 \mathrm{e}-15$ & 3.800 & $\ldots$ & 3.800 & 1 & 0.95 \\
\hline 1730 & CXOCJ100152.3+023153 & $1.40 \mathrm{e}-15$ & $3.89 \mathrm{e}-16$ & $-2.14 \mathrm{e}-15$ & $\ldots$ & $2.96 \mathrm{e}-15$ & $1.18 \mathrm{e}-15$ & 4.450 & $\ldots$ & 4.450 & 1 & 1.00 \\
\hline 1818 & CXOCJ100139.4+022152 & $2.97 \mathrm{e}-16$ & $1.30 \mathrm{e}-16$ & $-1.99 \mathrm{e}-15$ & $\ldots$ & $6.89 \mathrm{e}-16$ & $4.40 \mathrm{e}-16$ & 3.270 & $\ldots$ & 2.423 & -1 & 0.29 \\
\hline 2220 & CXOCJ095908.1+022707 & $7.29 \mathrm{e}-16$ & $2.18 \mathrm{e}-16$ & $-3.61 e-15$ & $\ldots$ & $2.09 \mathrm{e}-15$ & $6.14 \mathrm{e}-16$ & 5.07 & 5.07 & 4.277 & 1 & 1 \\
\hline 2378 & CXOCJ100048.8+021448 & $4.95 \mathrm{e}-16$ & $1.42 \mathrm{e}-16$ & $-4.72 \mathrm{e}-15$ & $\ldots$ & $1.30 \mathrm{e}-15$ & $4.67 \mathrm{e}-16$ & 3.433 & $\ldots$ & 2.195 & -1 & 0.23 \\
\hline 2518 & CXOCJ095905.0+022157 & $2.16 \mathrm{e}-16$ & $1.39 \mathrm{e}-16$ & $-5.30 \mathrm{e}-15$ & $\cdots$ & $1.29 \mathrm{e}-15$ & $5.89 \mathrm{e}-16$ & 3.446 & $\ldots$ & 3.446 & 1 & 0.97 \\
\hline 3299 & CXOCJ100120.3+014718 & $4.87 \mathrm{e}-16$ & $1.85 \mathrm{e}-16$ & $-1.24 \mathrm{e}-14$ & $\ldots$ & $2.61 \mathrm{e}-15$ & $7.03 e-16$ & 3.196 & .. & 2.680 & -1 & 0.35 \\
\hline 3373 & CXOCJ095946.6+014424 & $5.92 \mathrm{e}-16$ & $2.47 \mathrm{e}-16$ & $-7.67 e-15$ & $\ldots$ & $2.85 e-15$ & $9.59 \mathrm{e}-16$ & 3.686 & .. & 2.611 & -1 & 0.45 \\
\hline 3440 & CXOCJ095933.4+015757 & $3.56 \mathrm{e}-16$ & $1.30 \mathrm{e}-16$ & $-8.63 e-16$ & & $8.08 \mathrm{e}-16$ & $4.34 \mathrm{e}-16$ & 3.057 & .. & 3.057 & 1 & 0.28 \\
\hline 3561 & CXOCJ095829.8+015307 & $6.57 e-16$ & $2.64 \mathrm{e}-16$ & $-1.29 \mathrm{e}-14$ & & $3.41 \mathrm{e}-15$ & $1.02 \mathrm{e}-15$ & 3.765 & $\ldots$ & 2.264 & -1 & 0.63 \\
\hline 3636 & CXOCJ 1000 & & $1.91 \mathrm{e}-16$ & -8.4 & & & & & 3.189 & 3.105 & 1 & 1.00 \\
\hline 3651 & CXOCJ100042.3+023410 & $4.49 \mathrm{e}-16$ & $2.02 \mathrm{e}-16$ & $-8.05 e-15$ & & $1.79 \mathrm{e}-15$ & $7.69 \mathrm{e}-16$ & 3.144 & $\ldots$ & 3.144 & 1 & 0.40 \\
\hline 705 & CXOCJ095907.7+022817 & $-1.08 \mathrm{e}-15$ & $\ldots$ & $3.79 \mathrm{e}-15$ & $1.31 \mathrm{e}-15$ & $2.69 \mathrm{e}-15$ & $7.94 \mathrm{e}-16$ & 3.245 & $\ldots$ & 3.245 & 1 & 1.00 \\
\hline 1180 & CXOCJ100131.4+020558 & $-1.09 \mathrm{e}-15$ & $\ldots$ & $1.86 \mathrm{e}-15$ & $1.01 \mathrm{e}-15$ & $1.72 \mathrm{e}-15$ & $6.21 \mathrm{e}-16$ & 4.490 & $\ldots$ & 4.490 & 1 & 0.98 \\
\hline 1247 & CXOCJ100015.2+022518 & $-9.47 e-16$ & $\ldots$ & $1.68 \mathrm{e}-15$ & $8.52 \mathrm{e}-16$ & $1.37 \mathrm{e}-15$ & $5.24 \mathrm{e}-16$ & 3.084 & $\ldots$ & 3.084 & 1 & 0.51 \\
\hline 1285 & CXOCJ100125.5+021326 & $-1.20 \mathrm{e}-15$ & $\ldots$ & $2.08 \mathrm{e}-15$ & $8.05 \mathrm{e}-16$ & $1.41 \mathrm{e}-15$ & $4.69 \mathrm{e}-16$ & 3.043 & .. & 3.043 & 1 & 0.24 \\
\hline 1434 & CXOCJ100021.7+015547 & $-6.96 \mathrm{e}-16$ & $\ldots$ & $1.94 \mathrm{e}-15$ & $9.93 \mathrm{e}-16$ & $1.37 \mathrm{e}-15$ & $5.76 \mathrm{e}-16$ & 3.206 & $\ldots$ & 3.206 & 1 & 0.45 \\
\hline 1588 & CXOCJ100235.3+022505 & $-8.43 e-16$ & $\ldots$ & $1.44 \mathrm{e}-14$ & $4.33 e-15$ & $9.41 \mathrm{e}-15$ & $2.59 \mathrm{e}-15$ & 3.081 & $\ldots$ & 3.081 & 1 & 0.43 \\
\hline
\end{tabular}


Table 3

(Continued)

\begin{tabular}{|c|c|c|c|c|c|c|c|c|c|c|c|c|}
\hline Chid $^{\mathrm{a}}$ & IAU Name ${ }^{b}$ & $F_{0.5-2 \mathrm{keV}^{\mathrm{c}}}$ & Error & $F_{2-10 \mathrm{keV}^{\mathrm{c}}}$ & Error & $F_{0.5-10 \mathrm{keV}^{\mathrm{c}}}$ & Error & $z^{\mathrm{d}}$ & $z_{\text {spec }}{ }^{\mathrm{d}}$ & $z_{\text {phot }}{ }^{\mathrm{d}}$ & flag $^{\mathrm{e}}$ & $w^{f}{ }^{f}$ \\
\hline 1734 & CXOCJ100156.4+022448 & $-4.08 \mathrm{e}-16$ & $\ldots$ & $1.54 \mathrm{e}-15$ & $7.90 \mathrm{e}-16$ & $8.84 \mathrm{e}-16$ & $4.33 e-16$ & 3.062 & $\ldots$ & 2.949 & -1 & 0.39 \\
\hline 2212 & CXOCJ095858.2+023207 & $-5.73 e-16$ & $\ldots$ & $4.25 \mathrm{e}-15$ & $1.52 \mathrm{e}-15$ & $2.84 \mathrm{e}-15$ & $9.05 e-16$ & 3.011 & $\ldots$ & 2.939 & -1 & 0.11 \\
\hline 641 & CXOCJ100156.7+022539 & $-1.33 e-15$ & $\ldots$ & $-1.09 \mathrm{e}-14$ & $\ldots$ & $1.97 \mathrm{e}-15$ & $5.95 e-16$ & 3.474 & $\ldots$ & 3.474 & 1 & 0.55 \\
\hline 2698 & CXOCJ100021.2+020306 & $-5.52 \mathrm{e}-16$ & $\ldots$ & $2.43 e-15$ & $9.51 \mathrm{e}-16$ & $1.47 \mathrm{e}-15$ & $5.48 \mathrm{e}-16$ & 3.009 & $\ldots$ & 3.009 & 1 & 0.04 \\
\hline 1163 & CXOCJ100039.3+021045 & $-1.13 \mathrm{e}-15$ & $\ldots$ & $-1.32 \mathrm{e}-14$ & $\ldots$ & $1.59 \mathrm{e}-15$ & $5.61 \mathrm{e}-16$ & 3.121 & $\ldots$ & 2.813 & -1 & 0.33 \\
\hline 3285 & CXOCJ100102.8+014354 & $-1.44 \mathrm{e}-15$ & $\ldots$ & $-8.46 \mathrm{e}-15$ & $\ldots$ & $2.03 e-15$ & $1.00 \mathrm{e}-15$ & 3.453 & $\ldots$ & 3.453 & 1 & 0.63 \\
\hline 3450 & CXOCJ095938.6+015033 & $-1.13 e-15$ & $\ldots$ & $-4.41 \mathrm{e}-15$ & $\ldots$ & $1.20 \mathrm{e}-15$ & $5.94 \mathrm{e}-16$ & 3.294 & $\ldots$ & 3.294 & 1 & 0.69 \\
\hline 3456 & CXOCJ095942.5+015059 & $-8.72 \mathrm{e}-16$ & $\ldots$ & $-4.07 e-15$ & $\ldots$ & $9.53 e-16$ & $4.81 \mathrm{e}-16$ & 3.429 & $\ldots$ & 3.429 & 1 & 1.00 \\
\hline 11633 & CXOCJ095935.4+021926 & $5.74 \mathrm{e}-16$ & $1.80 \mathrm{e}-16$ & $5.80 \mathrm{e}-15$ & $\ldots$ & $-1.05 \mathrm{e}-14$ & $\ldots$ & 3.163 & $\ldots$ & 3.163 & 1 & 0.55 \\
\hline 325 & CXOCJ095840.6+021003 & $1.77 \mathrm{e}-15$ & $3.37 \mathrm{e}-16$ & $7.59 \mathrm{e}-15$ & $1.58 \mathrm{e}-15$ & $8.28 \mathrm{e}-15$ & $1.16 \mathrm{e}-15$ & 3.089 & 3.089 & 3.094 & 1 & 1.00 \\
\hline 688 & CXOCJ100122.8+015728 & $9.20 \mathrm{e}-16$ & $2.06 \mathrm{e}-16$ & $5.01 \mathrm{e}-15$ & $1.22 \mathrm{e}-15$ & $4.85 e-15$ & $7.96 \mathrm{e}-16$ & 3.430 & $\ldots$ & 2.877 & -1 & 0.74 \\
\hline 879 & CXOCJ100132.0+020428 & $2.49 \mathrm{e}-16$ & $1.34 \mathrm{e}-16$ & $2.70 \mathrm{e}-15$ & $1.01 \mathrm{e}-15$ & $1.92 \mathrm{e}-15$ & $6.00 \mathrm{e}-16$ & 3.852 & $\ldots$ & 3.852 & 1 & 0.97 \\
\hline 964 & CXOCJ100108.3+021834 & $1.08 \mathrm{e}-15$ & $1.87 \mathrm{e}-16$ & $2.49 \mathrm{e}-15$ & $9.36 \mathrm{e}-16$ & $3.73 e-15$ & $6.16 \mathrm{e}-16$ & 3.210 & $\ldots$ & 2.360 & -1 & 0.29 \\
\hline 965 & CXOCJ100036.5+021828 & $3.27 \mathrm{e}-16$ & $1.18 \mathrm{e}-16$ & $3.71 \mathrm{e}-15$ & $8.82 \mathrm{e}-16$ & $2.76 \mathrm{e}-15$ & $5.35 e-16$ & 3.175 & 3.175 & 3.160 & 1 & 1.00 \\
\hline 1263 & CXOCJ100142.0+021843 & $2.73 e-16$ & $1.14 \mathrm{e}-16$ & $1.96 \mathrm{e}-15$ & $7.44 \mathrm{e}-16$ & $1.61 \mathrm{e}-15$ & $4.59 \mathrm{e}-16$ & 3.092 & $\ldots$ & 3.092 & 1 & 0.62 \\
\hline 1269 & CXOCJ100211.1+021326 & $2.75 \mathrm{e}-15$ & $4.02 \mathrm{e}-16$ & $1.02 \mathrm{e}-14$ & $1.99 \mathrm{e}-15$ & $1.23 \mathrm{e}-14$ & $1.43 e-15$ & 3.440 & $\ldots$ & 3.440 & 1 & 0.75 \\
\hline 1276 & CXOCJ100119.9+022629 & $1.44 \mathrm{e}-15$ & $2.76 \mathrm{e}-16$ & $1.92 \mathrm{e}-15$ & $1.14 \mathrm{e}-15$ & $5.39 \mathrm{e}-15$ & $9.24 \mathrm{e}-16$ & 3.018 & $\ldots$ & 3.018 & 1 & 0.41 \\
\hline 315 & CXOCJ095910.3+020641 & $9.96 \mathrm{e}-16$ & $1.91 \mathrm{e}-16$ & $-1.31 \mathrm{e}-14$ & & $3.87 e-15$ & $6.47 \mathrm{e}-16$ & 3.006 & $\ldots$ & 2.982 & -1 & 0.00 \\
\hline 784 & CXOCJ100112.1+021802 & $3.09 \mathrm{e}-16$ & $1.52 \mathrm{e}-16$ & $-6.74 \mathrm{e}-15$ & $\ldots$ & $1.37 \mathrm{e}-15$ & $6.19 \mathrm{e}-16$ & 3.498 & 3.498 & 3.441 & 1 & 1.00 \\
\hline 871 & CXOCJ100023.9+020908 & $7.62 \mathrm{e}-16$ & $1.64 \mathrm{e}-16$ & $-6.56 \mathrm{e}-15$ & $\ldots$ & $2.09 \mathrm{e}-15$ & $4.85 e-16$ & 3.452 & $\ldots$ & 3.452 & 1 & 0.33 \\
\hline 1303 & CXOCJ095957.7+021750 & $8.96 \mathrm{e}-16$ & $1.91 \mathrm{e}-16$ & $-8.42 \mathrm{e}-15$ & $\ldots$ & $3.43 e-15$ & $6.68 \mathrm{e}-16$ & 3.026 & 3.026 & 3.027 & 1 & 1.00 \\
\hline 3293 & CXOCJ100113.4+014541 & $2.90 \mathrm{e}-16$ & $1.67 \mathrm{e}-16$ & $-8.76 \mathrm{e}-15$ & $\ldots$ & $1.72 \mathrm{e}-15$ & $6.75 e-16$ & 3.260 & $\ldots$ & 3.260 & 1 & 0.84 \\
\hline 735 & CXOCJ100015.2+014638 & $-1.47 \mathrm{e}-15$ & $\ldots$ & $2.23 \mathrm{e}-15$ & $9.07 e-16$ & $2.01 \mathrm{e}-15$ & $5.91 \mathrm{e}-16$ & 3.720 & $\ldots$ & 3.720 & 1 & 0.52 \\
\hline 1134 & CXOCJ095932.6+021633 & $-1.10 \mathrm{e}-15$ & $\ldots$ & $1.09 \mathrm{e}-15$ & $6.94 \mathrm{e}-16$ & $1.28 \mathrm{e}-15$ & $4.54 \mathrm{e}-16$ & 3.335 & 3.335 & 3.321 & 1 & 1.00 \\
\hline 2177 & CXOCJ095951.9+022556 & $-8.70 \mathrm{e}-16$ & $\ldots$ & $3.51 \mathrm{e}-15$ & $9.79 \mathrm{e}-16$ & $2.08 \mathrm{e}-15$ & $5.50 \mathrm{e}-16$ & 3.383 & $\ldots$ & 3.383 & 1 & 0.55 \\
\hline 2550 & CXOCJ095929.8+022141 & $2.24 \mathrm{e}-16$ & $1.16 \mathrm{e}-16$ & $-5.88 \mathrm{e}-15$ & $\ldots$ & $8.83 e-16$ & $4.43 e-16$ & 6.800 & $\ldots$ & 6.800 & 1 & 0.59 \\
\hline 1514 & CXOCJ100020.6+020819 & $3.25 \mathrm{e}-16$ & $1.24 \mathrm{e}-16$ & $2.50 \mathrm{e}-15$ & $8.41 \mathrm{e}-16$ & $2.22 \mathrm{e}-15$ & $5.29 \mathrm{e}-16$ & 5.113 & $\ldots$ & 5.113 & 1 & 0.82 \\
\hline 125 & CXOCJ100051.5+023457 & $8.51 \mathrm{e}-16$ & $2.62 \mathrm{e}-16$ & $-7.01 \mathrm{e}-15$ & $\ldots$ & $2.41 \mathrm{e}-15$ & $7.98 \mathrm{e}-16$ & 5.300 & 5.300 & 5.380 & 1 & 1.00 \\
\hline 3336 & CXOCJ100032.3+014305 & $-6.67 e-16$ & $\ldots$ & $-5.61 \mathrm{e}-15$ & $\ldots$ & $1.07 \mathrm{e}-15$ & $7.45 \mathrm{e}-16$ & 5.160 & $\ldots$ & 1.470 & -1 & 0.43 \\
\hline 75 & CXOCJ100059.4+022632 & $2.26 \mathrm{e}-15$ & $2.55 \mathrm{e}-16$ & $1.25 \mathrm{e}-14$ & $1.40 \mathrm{e}-15$ & $1.21 \mathrm{e}-14$ & $9.54 \mathrm{e}-16$ & 3.029 & 3.029 & 2.973 & 1 & 1.00 \\
\hline 472 & CXOCJ095952.6+021817 & $1.34 \mathrm{e}-15$ & $2.40 \mathrm{e}-16$ & $6.83 e-15$ & $1.40 \mathrm{e}-15$ & $7.05 \mathrm{e}-15$ & $9.30 \mathrm{e}-16$ & 3.155 & 3.155 & $\ldots$ & 1 & 1.00 \\
\hline 931 & CXOCJ100126.3+020424 & $7.65 \mathrm{e}-16$ & $1.73 e-16$ & $-5.13 e-15$ & $\ldots$ & $2.11 \mathrm{e}-15$ & $5.41 \mathrm{e}-16$ & 4.917 & 4.917 & $\ldots$ & 1 & 1.00 \\
\hline 1976 & CXOCJ095948.5+023905 & $-5.87 \mathrm{e}-16$ & $\ldots$ & $2.86 e-15$ & $1.28 \mathrm{e}-15$ & $1.77 \mathrm{e}-15$ & $7.32 \mathrm{e}-16$ & 3.080 & 3.080 & $\ldots$ & 1 & 1.00 \\
\hline 386 & CXOCJ100130.9+015234 & $8.52 \mathrm{e}-16$ & $2.48 \mathrm{e}-16$ & $2.71 \mathrm{e}-15$ & $1.20 \mathrm{e}-15$ & $3.65 \mathrm{e}-15$ & $8.69 \mathrm{e}-16$ & 3.240 & $\ldots$ & 3.240 & 1 & 0.37 \\
\hline 841 & CXOCJ100006.1+020013 & $-1.21 \mathrm{e}-15$ & $\ldots$ & $1.65 \mathrm{e}-15$ & $7.97 \mathrm{e}-16$ & $1.44 \mathrm{e}-15$ & $4.89 \mathrm{e}-16$ & 3.120 & $\ldots$ & 3.120 & 1 & 0.39 \\
\hline
\end{tabular}

Notes.

a X-ray identifier number from the C-COSMOS catalog (Elvis et al. 2009).

b Official IAU designation for the C-COSMOS sources.

c X-ray fluxes and flux errors from the Elvis et al. (2009) catalog. Negative numbers represent upper limits on the flux.

${ }^{\mathrm{d}}$ Chosen redshift; spectroscopic redshift; photometric redshift.

${ }^{\mathrm{e}}$ Redshift flag: when flag $=-1$ it means the source has been included in the sample because of its broad $P(z)$ and the redshift listed is equal to $z$ phot $+1 \sigma$.

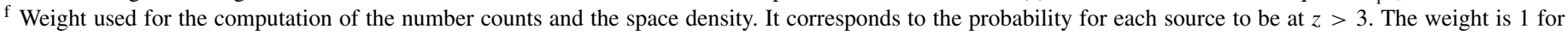
sources with spectroscopic redshift.

The number counts have been derived in the soft $0.5-2 \mathrm{keV}$ band (observed), while the space density has been computed in the hard 2-10 keV band (rest frame) to minimize the bias introduced by the obscuration. Errors associated with the uncertainties on the sensitivity of the survey and the assumption on the X-ray spectral shape are taken into account.

Studying the number counts of high-redshift quasars can constrain the evolution of the $\mathrm{BH}$ properties. Comparing physical models with number counts could be more efficient than comparing it directly with the luminosity function, which is a derived quantity that is affected by the selection function. The observed number counts at $z>3$ and $z>4$ are better reproduced by models with a prolonged quasar light curve characterized by a long, post-peak activity phase (dot-dashed line in Figure 3; Shankar 2010), and a higher minimum halo mass hosting quasars with respect to a model with smaller halo mass (e.g., Shen 2009; dashed line in Figure 3). Alternatively, we found that a model characterized by lower minimum host halo masses and negligi- ble post-peak phase can reproduce the number counts equally well (dotted line in Figure 3). A combined analysis with number counts and clustering measurements (e.g., Allevato et al. 2011) in this redshift and luminosity range should help to break these physical model degeneracies.

Taking advantage of the large number of sources and the depth of the survey, we are able to probe the space density of X-ray-selected quasars up to $z \sim 5$ at high luminosity $\left(\log L_{(2-10 \mathrm{keV})}>44.15 \mathrm{erg} \mathrm{s}^{-1}\right)$. The combination of soft-, hard-, and full-band-detected sources allows us to account for the presence of highly obscured sources in the derivation of the space density with limited model assumptions.

The comoving space density is in agreement with the predictions from the LDDE Gilli et al. (2007) model at high X-ray luminosity and at all redshifts, confirming the declining space density as observed in the optical. Doubling the sample, at least, in the $z>5$ and $z>6$ bins would strengthen the agreement with the Gilli et al. (2007) model, now based on a sample of 


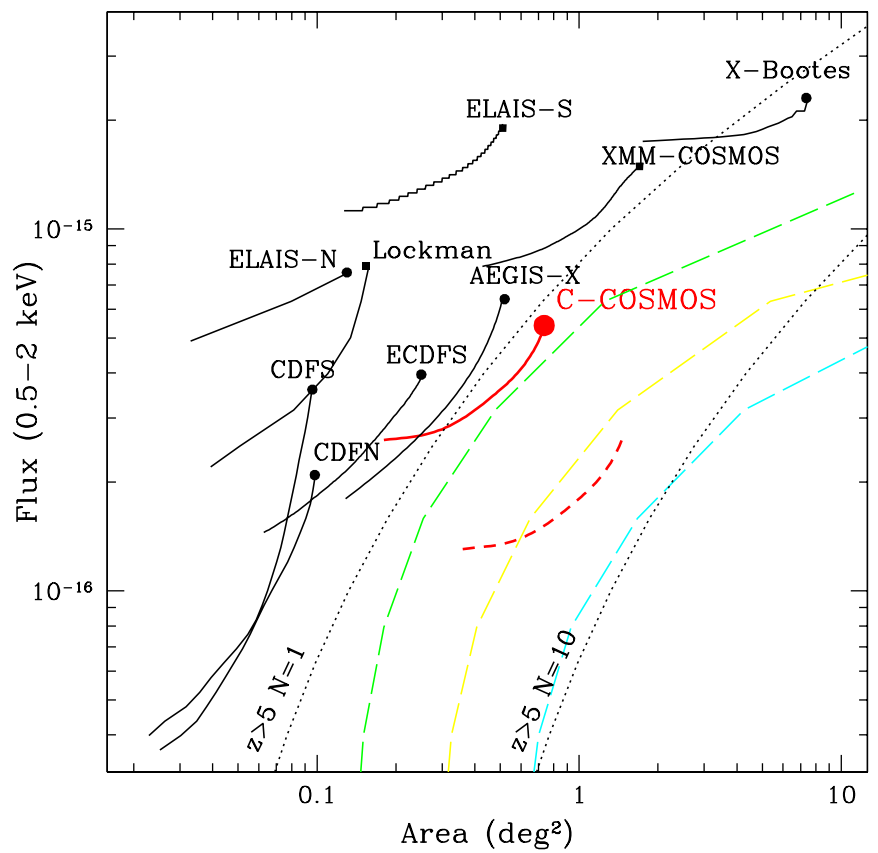

Figure 5. Area-flux curves for Chandra and XMM-Newton contiguous X-ray surveys (black solid lines). Each survey has been plotted using each sensitivity curve starting from the flux corresponding to the area that is $80 \%$ of the maximum area for that survey (large points at the top of each curve), to the flux corresponding to the $20 \%$ of the total area (bottom of each curve). For the references of each survey see Figure 5 of Elvis et al. (2009). The red solid and dashed lines represent the C-COSMOS survey and a potential 6 Ms large Chandra COSMOS survey (see the text), respectively. The dashed lines show the area and flux required to observe 15 sources with luminosity in the range $10^{43}-10^{44} \mathrm{erg} \mathrm{s}^{-1}$, in the following redshift bins: $z=3-3.5$, green dashed; $z=$ $3.5-4$, yellow dashed; $z=4-4.5$, cyan dashed. The dotted lines show the area and flux required to observe $N=1,10$ bright X-ray luminosity $\left(>10^{44} \mathrm{erg} \mathrm{s}^{-1}\right)$ quasars at $z>5$.

(A color version of this figure is available in the online journal.)

five sources. The LADE Aird et al. (2010) model overestimates the space density at $z>4$, even when the errors are taken into account.

The flux limit of the survey, thus the lack of sources beyond $z>3.5$ in the low-luminosity regime, does not yet allow strong constraints to be put on the density evolution at the faint end $\left(\log L_{(2-10 \mathrm{keV})}<44.15 \mathrm{erg} \mathrm{s}^{-1}\right)$, where somewhat different results have been recently found in the optical band by Glikman et al. (2010, 2011) and Ikeda et al. (2011), with respect to previous studies (e.g., Fontanot et al. 2007). Despite our sample of 32 sources at $3<z<3.5$ in the low-luminosity range, it is not possible to give firm results when comparing the data with models. To obtain a more conclusive understanding of the quasar evolution in the early universe, in particular at $z>3.5$, where the models diverge strongly, larger and complete samples of low-luminosity AGNs are required.

We can now quantify the best strategy to obtain these larger samples. In Figure 5, the well-known "area-flux" plot (e.g., Brandt \& Hasinger 2005) used to compare different X-ray surveys is reported as in Elvis et al. (2009; i.e., plotting the flux at which each survey reaches from $20 \%$ to $80 \%$ of the area surveyed).

Using the agreement of the predictions between the Gilli et al. (2007) model with our data, we estimated curves of a constant number of sources $(N=15)$ in three redshift bins $(z=3-3.5$ green dashed; $z=3.5-4$ yellow dashed; $z=4-4.5$ cyan dashed) for the low-luminosity range $10^{43}-10^{44} \mathrm{erg} \mathrm{s}^{-1}$, to estimate the flux limit and the area needed to produce larger sample of faint AGNs at high redshift. These curves show that moving the survey sensitivity diagonally in this plot is a better investment, for these purposes than moving parallel to the axes for either increased depth or area.

Doubling the coverage of the COSMOS area and increasing the depth by a factor two (dashed red thick line) would substantially increase the $z>3$ low-luminosity AGN statistics by a factor two in the $10^{43}-10^{44} \mathrm{erg} \mathrm{s}^{-1}$ range. This would be sufficient to resolve the controversial optical results in this luminosity range, based on small samples.

A "double-doubled" survey of this kind would also increase the sample of $z>5$ quasars (dotted black line) from four in the soft band to seven to eight sources at high luminosity. Such a survey, performed with the same observational strategy of CCOSMOS (i.e., homogeneous exposure time and same tiling), would take approximately $6 \mathrm{Ms}$ with Chandra, the scale of the $\mathrm{X}$-ray Visionary Projects. In this respect, the proposed Wide Field X-ray Telescope (WFXT) Medium survey (Rosati et al. 2011) designed to cover a large area $\left(3000 \mathrm{deg}^{2}\right)$ at the fluxes of the C-COSMOS survey would be optimal to collect order of magnitude larger samples of high-redshift AGNs compared to X-ray and also optical surveys now available (Brusa et al. 2011; Gilli et al. 2011b).

The authors thank the referee for suggestions which improved this paper's content. The authors thank J. Aird and E. Glikman for sharing their luminosity functions, and G. Risaliti, R. D'Abrusco, and A. Goulding for useful discussions. This work was supported in part by NASA Chandra grant No. GO7-8136A (M.E., F.C., H.H.), the Blancheflor Boncompagni Ludovisi foundation (F.C.) and the Smithsonian Scholarly Studies (F.C.). F.S. acknowledges the Alexander von Humboldt Foundation for support. In Italy this work is supported by ASI/INAF contracts $\mathrm{I} / 009 / 10 / 0$ and I/088/06.

\section{Facilities: CXO (ACIS), HST (ACS), XMM}

\section{REFERENCES}

Aird, J., Nandra, K., Laird, E. S., et al. 2010, MNRAS, 401, 2531

Allevato, V., Finoguenov, A., Cappelluti, N., et al. 2011, ApJ, 736, 99

Arnaud, K. A. 1996, in ASP Conf. Ser. 101, Astronomical Data Analysis Software and Systems V, ed. G. H. Jacoby \& J. Barnes (San Francisco, CA: ASP), 17

Avni, Y., \& Bahcall, J. N. 1980, ApJ, 235, 694

Barger, A. J., Cowie, L. L., Mushotzky, R. F., et al. 2005, AJ, 129, 578

Brandt, W. N., \& Hasinger, G. 2005, ARA\&A, 43, 827

Brusa, M., Civano, F., Comastri, A., et al. 2010, ApJ, 716, 348

Brusa, M., Comastri, A., Gilli, R., et al. 2009, ApJ, 693, 8

Brusa, M., Gilli, R., Civano, F., et al. 2011, Mem. Soc. Astron. Ital. Suppl., 17, 106

Capak, P. L., Riechers, D., Scoville, N. Z., et al. 2011, Nature, 470, 233

Cassata, P., Le Fèvre, O., Garilli, B., et al. 2011, A\&A, 525, A143

Comastri, A., Ranalli, P., Iwasawa, K., et al. 2011, A\&A, 526, L9

Croton, D. J., Springel, V., White, S. D. M., et al. 2006, MNRAS, 365, 11

Ebrero, J., Carrera, F. J., Page, M. J., et al. 2009, A\&A, 493, 55

Elvis, M., Civano, F., Vignali, C., et al. 2009, ApJS, 184, 158

Fan, X., Narayanan, V. K., Lupton, R. H., et al. 2001, AJ, 122, 2833

Fiore, F. 2010, in AIP Conf. Proc. 1248, X-Ray Astronomy 2009: Present Status, Multi-wavelength Approach and Future Perspectives, ed. A. Comastri, L. Angelini, \& M. Cappi (Melville, NY: AIP), 373

Fontanot, F., Cristiani, S., Monaco, P., et al. 2007, A\&A, 461, 39

Gallerani, S., Maiolino, R., Juarez, Y., et al. 2010, A\&A, 523, A85

Gilli, R., Comastri, A., \& Hasinger, G. 2007, A\&A, 463, 79

Gilli, R., Su, J., Norman, C., et al. 2011a, ApJ, 730, L28

Gilli, R., Tozzi, P., Rosati, P., et al. 2011b, Mem. Soc. Astron. Ital. Suppl., 17, 85

Glikman, E., Bogosavljević, M., Djorgovski, S. G., et al. 2010, ApJ, 710, 1498

Glikman, E., Djorgovski, S. G., Stern, D., et al. 2011, ApJ, 728, L26 
Granato, G. L., De Zotti, G., Silva, L., Bressan, A., \& Danese, L. 2004, ApJ 600,580

Granato, G. L., Silva, L., Monaco, P., et al. 2001, MNRAS, 324, 757

Hasinger, G., Miyaji, T., \& Schmidt, M. 2005, A\&A, 441, 417

Hasinger, G. 2008, A\&A, 490, 905

Hopkins, P. F., Hernquist, L., Cox, T. J., et al. 2006, ApJS, 163, 1

Ikeda, H., Nagao, T., Matsuoka, K., et al. 2011, ApJ, 728, L25

Jiang, L., Fan, X., Hines, D. C., et al. 2006, AJ, 132, 2127

Koekemoer, A. M., Alexander, D. M., Bauer, F. E., et al. 2004, ApJ, 600, L123

Lapi, A., Shankar, F., Mao, J., et al. 2006, ApJ, 650, 42

Lilly, S. J., Le Brun, V., Maier, C., et al. 2009, ApJS, 184, 218

Lilly, S. J., Le Fèvre, O., Renzini, A., et al. 2007, ApJS, 172, 70

Luo, B., Brandt, W. N., Xue, Y. Q., et al. 2010, ApJS, 187, 560

Marshall, H. L., Tananbaum, H., Avni, Y., \& Zamorani, G. 1983, ApJ, 269, 35

Menci, N., Fiore, F., Puccetti, S., \& Cavaliere, A. 2008, ApJ, 686, 219

Murayama, T., Taniguchi, Y., Scoville, N. Z., et al. 2007, ApJS, 172, 523

Nandra, K., \& Pounds, K. A. 1994, MNRAS, 268, 405

Norman, C., Hasinger, G., Giacconi, R., et al. 2002, ApJ, 571, 218

Puccetti, S., Vignali, C., Cappelluti, N., et al. 2009, ApJS, 185, 586

Richards, G. T., Strauss, M. A., Fan, X., et al. 2006, AJ, 131, 2766

Rosati, P., Borgani, S., Gilli, R., et al. 2011, Mem. Soc. Astron. Ital. Suppl., 17, 8

Salvato, M., Hasinger, G., Ilbert, O., et al. 2009, ApJ, 690, 1250
Schmidt, M. 1968, ApJ, 151, 393

Schmidt, M., Schneider, D. P., \& Gunn, J. E. 1995, AJ, 110, 68

Shankar, F. 2009, New Astron. Rev., 53, 57

Shankar, F. 2010, in IAU Symp. 267, Co-evolution of Central Black Holes and Galaxies, ed. B. Peterson, R. Somerville, \& T. Storchi-Bergmann (Cambridge: Cambridge Univ. Press), 248

Shankar, F., Crocce, M., Miralda-Escudé, J., Fosalba, P., \& Weinberg, D. H. 2010, ApJ, 718, 231

Shapley, A. E., Steidel, C. C., Pettini, M., \& Adelberger, K. L. 2003, ApJ, 588, 65

Shen, Y. 2009, ApJ, 704, 89

Silverman, J. D., Green, P. J., Barkhouse, W. A., et al. 2005, ApJ, 624, 630

Silverman, J. D., Green, P. J., Barkhouse, W. A., et al. 2008, ApJ, 679, 118

Silverman, J. D., Mainieri, V., Salvato, M., et al. 2010, ApJS, 191, 124

Steffen, A. T., Barger, A. J., Capak, P., et al. 2004, AJ, 128, 1483

Trump, J. R., Impey, C. D., Elvis, M., et al. 2009, ApJ, 696, 1195

Trump, J. R., Impey, C. D., McCarthy, P. J., et al. 2007, ApJS, 172, 383

Willott, C. J., McLure, R. J., \& Jarvis, M. J. 2003, ApJ, 587, L15

Wyithe, J. S. B., \& Loeb, A. 2003, ApJ, 595, 614

Xue, Y. Q., Luo, B., Brandt, W. N., et al. 2011, ApJS, 195, 10

Yencho, B., Barger, A. J., Trouille, L., \& Winter, L. M. 2009, ApJ, 698, 380

Young, M., Elvis, M., \& Risaliti, G. 2010, ApJ, 708, 1388 